\title{
The Behaviour of Mistuned Piezoelectric Shunt Systems and Its Estimation
}

\author{
M. Berardengo, ${ }^{1}$ S. Manzoni, ${ }^{2}$ and M. Vanali $^{1}$ \\ ${ }^{1}$ Department of Industrial Engineering, Università degli Studi di Parma, Parco Area delle Scienze 181/A, 43124 Parma, Italy \\ ${ }^{2}$ Department of Mechanical Engineering, Politecnico di Milano, Via La Masa 34, 20156 Milan, Italy \\ Correspondence should be addressed to S. Manzoni; stefano.manzoni@polimi.it
}

Received 20 May 2016; Revised 18 July 2016; Accepted 26 July 2016

Academic Editor: Nicola Caterino

Copyright (c) 2016 M. Berardengo et al. This is an open access article distributed under the Creative Commons Attribution License, which permits unrestricted use, distribution, and reproduction in any medium, provided the original work is properly cited.

\begin{abstract}
This paper addresses monoharmonic vibration attenuation using piezoelectric transducers shunted with electric impedances consisting of a resistance and an inductance in series. This type of vibration attenuation has several advantages but suffers from problems related to possible mistuning. In fact, when either the mechanical system to be controlled or the shunt electric impedance undergoes a change in their dynamical features, the attenuation performance decreases significantly. This paper describes the influence of biases in the electric impedance parameters on the attenuation provided by the shunt and proposes an approximated model for a rapid prediction of the vibration damping performance in mistuned situations. The analytical and numerical results achieved within the paper are validated using experimental tests on two different test structures.
\end{abstract}

\section{Introduction}

Vibration attenuation in light structures is a widely studied topic and often takes advantage of the use of smart materials, which are characterised by useful properties. Indeed, these materials are inexpensive when compared to other control systems, and they are characterised by low weight. This last feature is a fundamental aspect because it avoids introducing high load effects on the controlled structure. Among smart materials, piezoelectric elements (particularly piezoelectric laminates, which are used in this paper) are among the best materials to attenuate vibrations in bidimensional (e.g., plates) and monodimensional (e.g., beams) structures [13]. There are several control techniques for light structures that rely on this type of actuator, and one of the most attractive is the shunt of the piezoelectric element. In this case, a properly designed electrical network is shunted to the piezoelectric bender bonded to the structure. The ability of the piezoelectric element to convert mechanical energy into electrical energy and vice versa $[4,5]$ is used, which allows a passive attenuation of the structure's vibration. This method was initially proposed by Hagood and von Flotow [4]. This technique is extremely attractive because it is cheap, it does not introduce energy into the system, that is, it cannot lead to instability, and it does not require any feedback signal.

When a monoharmonic control is required, the most effective shunt electric impedance consists of a resistance $R$ and an inductance $L$ in series $[2,4,6-8]$ (resonant shunt or $R L$ shunt). These two elements, along with the capacitance $C_{p}$ of the piezoelectric actuator (i.e., the piezoelectric actuator is modelled here as a capacitance and a strain-induced voltage generator in series; see Section 2), constitute a resonant circuit, which is the electric equivalent of the mechanical tuned mass damper (TMD) [2]. Therefore, this circuit is able to damp the structural vibration corresponding to a given eigenmode as soon as its dynamic features are tuned to those of the vibrating structure.

There are several methods in the literature that explain how to select the values of $R$ and $L$ to optimise the vibration attenuation. Hagood and von Flotow [4] proposed two different tuning strategies based on considerations on the shape of the system transfer function and on the pole placement techniques for an undamped structure. Both these tuning methods are based on the classical TMD theory. Høgsberg and Krenk $[9,10]$ developed another calibration method based on the pole placement for $R L$ circuits in series and 
parallel. The values of $R$ and $L$ are selected to guarantee equal modal damping of the two modes of the electromechanical structure and good separation of the complex poles. Thomas et al. [11] proposed two different methods, even for damped structures, that relied on the transfer function criteria and pole placement and provided closed formulas to estimate the attenuation performance.

Although all of the mentioned tuning strategies work extremely well, one significant issue of shunt damping using $R L$ impedances is that this type of electrical circuit is not adaptive. This in turn means that it is not possible to follow possible changes in the dynamic behaviour of either the vibrating structure (e.g., a temperature shift can change the eigenfrequency of the mode to be controlled) or the impedance itself (e.g., a temperature shift can cause a significant change in the $R$ value [12]). Hence, this control technique often works in mistuned conditions, even when starting from a perfect tuning condition. This mistuning leads to severe worsening of the attenuation performance.

A few techniques based on adaptive circuits were proposed to overcome the limitations due to uncertainties in the mechanical and electrical quantities. Based on the singlemode control, Hollkamp and Starchville developed a selftuning $R L$ circuit that was able to follow any change in the frequency of the mode to be controlled [13]. This technique is based on a synthetic circuit (which provides both the resistance and the inductance) consisting of two operational amplifiers and a motorised potentiometer. Despite its effectiveness, this method only considers a mistuning due to a change in the eigenfrequency to be controlled and does not consider other types of changes or uncertainties, such as ones related to electrical parameters. Furthermore, this method is active, thus losing the advantage provided by the passive shunt technique. Other recent studies by Zhou et al. [14, 15] attempted to determine methods to limit the problem of mistuning by using nonlinear elements when the disturbance was harmonic and using more than one piezoelectric actuator bonded to the vibrating structure. Although these techniques can be effectively employed, their use implies the loss of the two primary features of the resonant piezoelectric shunt: linearity (and thus ease of use) and passivity. Therefore, the analysis of the performances of traditional $R L$ shunts in mistuned conditions still has significant relevance.

Although the problems related to mistuning are evidenced in literature [16-18], there have been few analyses on shunt robustness. These analyses are of significant interest for numerous engineering applications where electrical power is often limited or even avoided, thus preventing the use of adaptation systems for the shunt impedance (e.g., space applications). In situations where passivity is requested, it is important to analyse the behaviour of the shunted system in the presence of mistuning because it worsens the attenuation performance. Recently, Berardengo et al. [19] studied the robustness of different optimisation methods for $R L$ circuits and determined the most robust method. Based on the outcomes of [19], this paper aims to further investigate the robustness of $R L$ shunt damping. The word robustness is intended here as the capability of the shunt impedance to attenuate the vibrations even when in mistuned conditions.
Therefore, this paper analyses the behaviour of mistuned electromechanical systems, thus depicting the relationship between the attenuation and the system parameters (e.g., coupling coefficient, mechanical nondimensional damping ratio, and eigenfrequency) in tuned and mistuned conditions. Furthermore, this paper demonstrates that the loss of attenuation primarily depends on only one bias (i.e., either the bias on the damping or the eigenfrequency of the electric resonant circuit) if the electrical damping is overestimated, whereas the effects of the two bias types (on the electrical eigenfrequency and damping) combine with each other when the electrical damping is underestimated. Based on these results, an approximated analytical model is proposed to estimate the attenuation performance with different amounts of mistuning using a small number of numerical simulations.

To summarise, the goals of this paper are to investigate how mistuned systems (which are often encountered in real applications) behave and consequently propose an approximated model that is able to predict the behaviour of the mistuned system with the least amount of numerical simulations. To reach the above goal, the authors highlight the relationship between the attenuation and all of the problem parameters and demonstrate that some of these relations can be approximated linearly in a logarithmic scale. Moreover, the authors bring to evidence the cases where the loss of performance depends on just one mistuning type (i.e., either the bias on the damping or the eigenfrequency of the electric resonant circuit), even though mistuning occurs on both, as well as the cases where both the mistuning types have an influence. All of these observations allow for the development of the mentioned approximated model for mistuned systems, which enhances the knowledge of their behaviour. Moreover, using this new simplified model, the authors demonstrate that an initially overestimated value of $R$ is able to decrease the loss in performance due to mistuning and explain why this phenomenon occurs. Additionally, this allows for guidelines to be provided on how to tune the shunt parameters when a mistuning is expected.

This paper is structured as follows. Section 2 discusses the model of the electromechanical system used in this paper. Section 3 highlights the linear relationship between the attenuation and the system parameters, which will be employed in Section 4 to analyse the effects of mistuning and propose an approximated model to describe the attenuation performance in the presence of mistuning. Lastly, Section 5 validates the previous results using experiments.

\section{Model of the Electromechanical System}

As mentioned in the previous section, the goal of this paper is to study the vibration attenuation of the controlled system in mistuned conditions. Thus, the most intuitive and used index to evaluate the attenuation performance is the ratio between the maximum of the dynamic amplification modulus in uncontrolled and controlled conditions [11, 19]. Therefore, for the performance analysis, it is necessary to derive the expression of the frequency response function of the electromechanical system and thus to introduce the model used to describe its electrodynamic behaviour. 


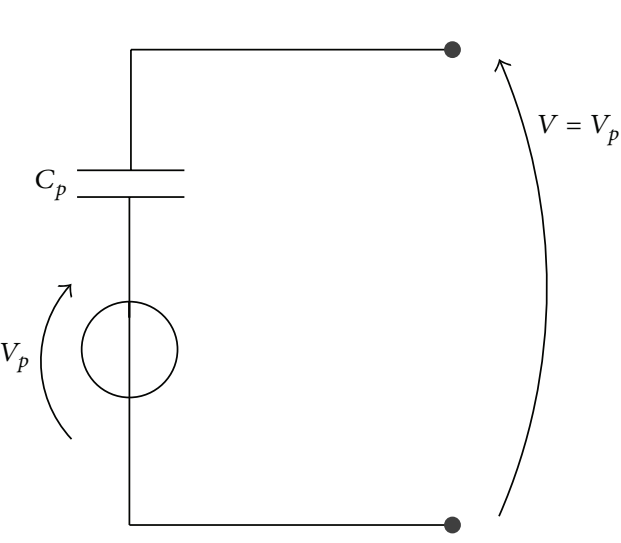

(a)

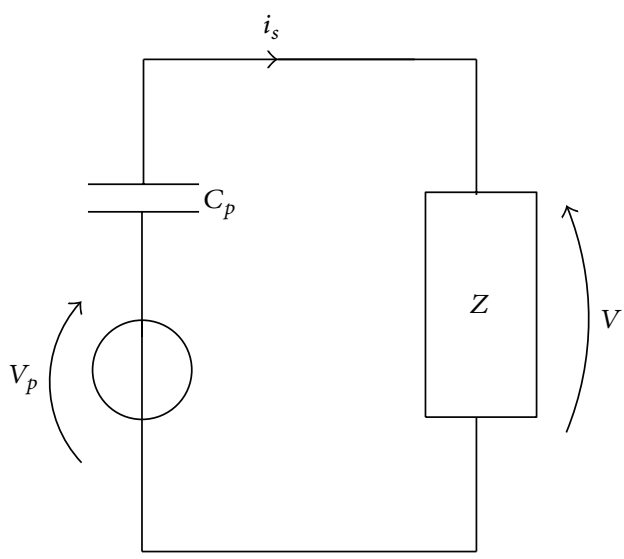

(b)

FIGURE 1: Electric equivalent of a piezoelectric actuator in open circuit (a) and shunted with impedance $Z$ (b).

The piezoelectric actuator is modelled here as a capacitance $C_{p}$ and a strain-induced voltage generator in series (Figure 1(a)). The induced voltage is $V_{p}$, whereas the voltage between the electrodes of the piezoelectric bender is $V$. $V$ is equal to $V_{p}$ when the piezoelectric actuator is opencircuited and null when the actuator is short-circuited. $V$ takes different values when an impedance $Z$ is shunted to the electrodes of the actuator (Figure 1(b)) because a current $i_{s}$ flows in the circuit. Moheimani et al. [20,21] proved that systems controlled by piezoelectric actuators shunted with electric impedances can be modelled as a double feedback loop (Figure 2(a)). The inner loop of Figure 2(a) can be observed as a controller $K$, which can be expressed in the Laplace domain as follows:

$$
K(s)=\frac{V}{V_{p}}=\frac{s C_{p} Z(s)}{1+s C_{p} Z(s)},
$$

where $s$ is the Laplace variable.

Because the shunt impedance $Z$ considered in this study is a resistance $R$ and an inductance $L$ (see Section 1) in series, $Z$ can be expressed in the Laplace domain as follows:

$$
Z(s)=L s+R
$$

The two terms $G_{v v}$ and $G_{v w}$ in Figure 2(a) are frequency response functions (FRFs). The former is the FRF between $V$ and $V_{p}$, whereas the latter is between a disturbance $W$ and $V_{p}$. These two FRFs can be expressed by the formulations in the Laplace domain [20] as follows:

$$
\begin{aligned}
G_{v v}(s) & =\frac{V_{p}}{V}=\gamma \sum_{n=1}^{\infty} \frac{\psi_{n} \psi_{n}}{s^{2}+2 \xi_{n} \omega_{n} s+\omega_{n}^{2}} \\
G_{v w}(s) & =\frac{V_{p}}{W}=\frac{\gamma}{\bar{K}} \sum_{n=1}^{\infty} \frac{\Phi_{n}\left(x_{F}\right) \psi_{n}}{s^{2}+2 \xi_{n} \omega_{n} s+\omega_{n}^{2}},
\end{aligned}
$$

where $\omega_{n}$ is the $n$th eigenfrequency of the structure with the piezoelectric bender short-circuited; $\xi_{n}$ is the associated nondimensional damping ratio; $\Phi_{n}$ is the $n$th eigenmode

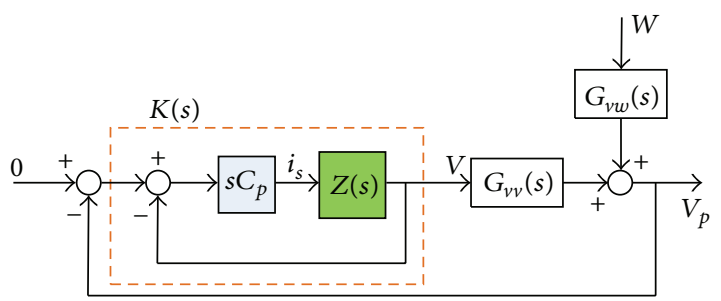

(a)

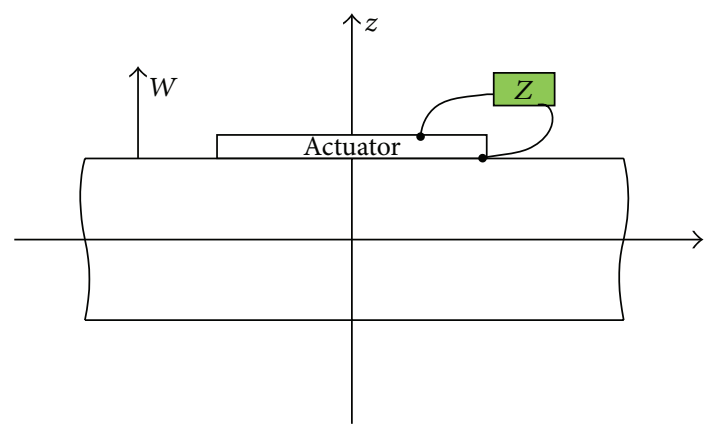

(b)

FIGURE 2: Feedback representation of the shunt control (a) and a structure subject to disturbance $W$ and damped using a piezoactuator shunted to an electric impedance $Z$ (b).

of the structure (scaled to the unit modal mass); $\Phi_{n}\left(x_{F}\right)$ represents the value of the $n$th mode at the forcing point $x_{F} ; \psi_{n}$ is a term depending on the curvature of the $n$th mode in the area of the piezoelectric patch $[20,21]$, which assumes different formulations for mono- and bidimensional structures; and $\gamma$ and $\bar{K}$ are two parameters based on the geometric, mechanical, and electrical features of the structure and the piezoelectric actuator. The method for calculating $\psi_{n}, \gamma$, and $\bar{K}$ for different possible configurations (e.g., monodimensional and bidimensional structures, symmetric and antisymmetric configuration of the piezoelectric actuator) can be found in [19]. 
The closed-loop FRF between disturbance $W$ (Figure $2(\mathrm{a}))$ and $V_{p}$ can be expressed as follows:

$$
T_{v w}(s)=\frac{V_{p}}{W}=\frac{G_{v w}(s)}{1+K(s) G_{v v}(s)} .
$$

Then, the closed-loop FRF between $W$ and the transverse displacement $z$ of the structure (Figure 2(b)) in a given point $x_{M}$, which describes the behaviour of the system damped by the shunt, can be expressed as follows:

$$
\begin{aligned}
\left.T_{z w}(s)\right|_{x=x_{M}} & =\frac{z\left(x_{M}\right)}{W}=\left.G(s)\right|_{x=x_{M}} \frac{T_{v w}(s)}{G_{v v}(s)} \\
& =\left.G(s)\right|_{x=x_{M}} \frac{G_{v w}(s)}{1+K(s) G_{v v}(s)} \cdot \frac{1}{G_{v v}(s)},
\end{aligned}
$$

where $G(s)$ is the FRF between $V$ and $z$ [19], which can be given as follows:

$$
G\left(s, x_{M}\right)=\frac{z\left(x_{M}\right)}{V}=\bar{K} \sum_{n=1}^{\infty} \frac{\Phi_{n}\left(x_{M}\right) \psi_{n}}{s^{2}+2 \xi_{n} \omega_{n} s+\omega_{n}^{2}} .
$$

Based on the aforementioned theoretical approach (see (5)), the formulation of $T_{z w}$ can be rearranged to achieve a compact expression. Thus, the eigenfrequency $\omega_{p}$ and the nondimensional damping ratio $d_{i}$ of the electric network (composed by the series of $C_{p}, L$, and $R$ ) [19] can be conveniently defined as follows:

$$
\begin{aligned}
& \omega_{p}=\frac{1}{\sqrt{L C_{p}}} \\
& d_{i}=\frac{R}{2} \sqrt{\frac{C_{p}}{L}} .
\end{aligned}
$$

By substituting (2), (7), and (8) into (1), the controller $K$ can be expressed as a function of these two quantities as follows:

$$
K=\frac{s\left(s+2 d_{i} \omega_{p}\right)}{s^{2}+2 d_{i} \omega_{p} s+\omega_{p}^{2}} .
$$

For single degree of freedom systems, the FRF $T_{z w}$ as a function of the electrical eigenfrequency and damping can be derived by substituting (9), (6), and (3) into (5) as follows:

$$
\begin{aligned}
& T_{z w}\left(s, x_{M}\right)=\Phi_{n}\left(x_{M}\right) \Phi_{n}\left(x_{F}\right) \\
& \cdot \frac{s^{2}+2 d_{i} \omega_{p} s+\omega_{p}^{2}}{\left(s^{2}+2 d_{i} \omega_{p} s+\omega_{p}^{2}\right)\left(s^{2}+2 \xi_{n} \omega_{n} s+\omega_{n}^{2}\right)+\gamma \psi_{n}^{2} s\left(s+2 d_{i} \omega_{p}\right)} .
\end{aligned}
$$

This formulation is valid for both beams and plates as well as for any layout of the piezoelectric actuator (e.g., single actuator, two colocated actuators) [19]. It should be noted that if the poles of this FRF are calculated considering the piezoelectric actuator in open-circuit condition $\left(i_{s}=0, Z=\right.$ $+\infty)$, then the expression of the open-circuit eigenfrequency $\omega_{n}^{\text {oc }}$ can be written as follows:

$$
\omega_{n}^{\mathrm{oc}}=\sqrt{\omega_{n}^{2}+\gamma \psi_{n}^{2}}
$$

Hence, it is possible to calculate the $n$th effective coupling factor $k_{n}$ (defined as $\sqrt{\left(\left(\omega_{n}^{\text {oc }}\right)^{2}-\omega_{n}^{2}\right) / \omega_{n}^{2}}$, e.g., $\left.[4,11,22]\right)$ using (11) as follows:

$$
k_{n}=\sqrt{\frac{\left(\omega_{n}^{\mathrm{oc}}\right)^{2}-\omega_{n}^{2}}{\omega_{n}^{2}}}=\frac{\sqrt{\gamma \psi_{n}^{2}}}{\omega_{n}} .
$$

It should be noted that $k_{n}$ does not depend on the type of shunt used but is a property of the system composed of the vibrating structure and the piezoelectric actuator; $k_{n}$ indicates the capability of the piezoelectric actuator, coupled to a given structure, to transform mechanical energy into electrical energy.

The performance of the controlled system in optimal conditions will depend on the tuning strategy selected to fix the values of $R$ and $L$. The one considered here is found as the most robust to possible mistuning in [19]. It is based on considerations on the shape of the FRF of (10). Nevertheless, it will be shown that the results and the procedure presented in this paper are valid for all tuning strategies that lead to a nearly flat shape of the FRF around the resonance frequency (see Section 3). The tuning criterion considered here fixes the values of $R$ and $L$ based on the procedure briefly summarised here below:

(i) The trend of $\left|T_{z w}\right|$ is independent of the damping factor of the electrical circuit $d_{i}$ at two frequency values $\omega_{A}$ and $\omega_{B}$ (see the corresponding points $A$ and $B$ in Figure 3(a)) ( $\omega$ is the circular frequency) for undamped systems [23]. The optimal value of $\omega_{p}$ $\left(\omega_{p}^{\mathrm{opt}}\right.$ ) can be found by imposing the same dynamic amplification modulus at these two frequencies. Thus, the expression for the electrical eigenfrequency can be achieved [19] as follows:

$$
\omega_{p}^{\mathrm{opt}}=\sqrt{\omega_{n}^{2}+\gamma \psi_{n}^{2}}=\omega_{n}^{\mathrm{oc}}
$$

Then, the value of $L$ can be found by combining (7) and (13).

(ii) The optimal value of the damping $d_{i}$ (and thus of $R$ ) is found by imposing an equal dynamic amplification $\left|T_{z w}\right|$ at two different frequencies: $\omega_{A}$ and a second frequency given by the square root of the arithmetic mean of $\omega_{A}^{2}$ and $\omega_{B}^{2}$. This frequency is found to be equal to the electrical frequency $\omega_{p}$ [19]. Thus, the condition used to fix the value of $d_{i}$ can be given as follows:

$$
\left|T_{z w}\right|_{\omega_{A}}=\left|T_{z w}\right|_{\omega_{p}}
$$

This condition is convenient to tune the shunt impedance because it allows a flat trend of $\left|T_{z w}\right|$ to occur in the frequency band around the resonance (Figure 3(b)). The value of the optimal electrical damping $\left(d_{i}^{\text {opt }}\right)$, which results from (14) (considering $\xi_{n}=0$ ), can be given as follows:

$$
d_{i, \xi_{n}=0}^{\mathrm{opt}}=\sqrt{\frac{\gamma \psi_{n}^{2}}{2\left(\omega_{p}^{\mathrm{opt}}\right)^{2}}} .
$$

Then, the value of $R$ can be found using (8). 


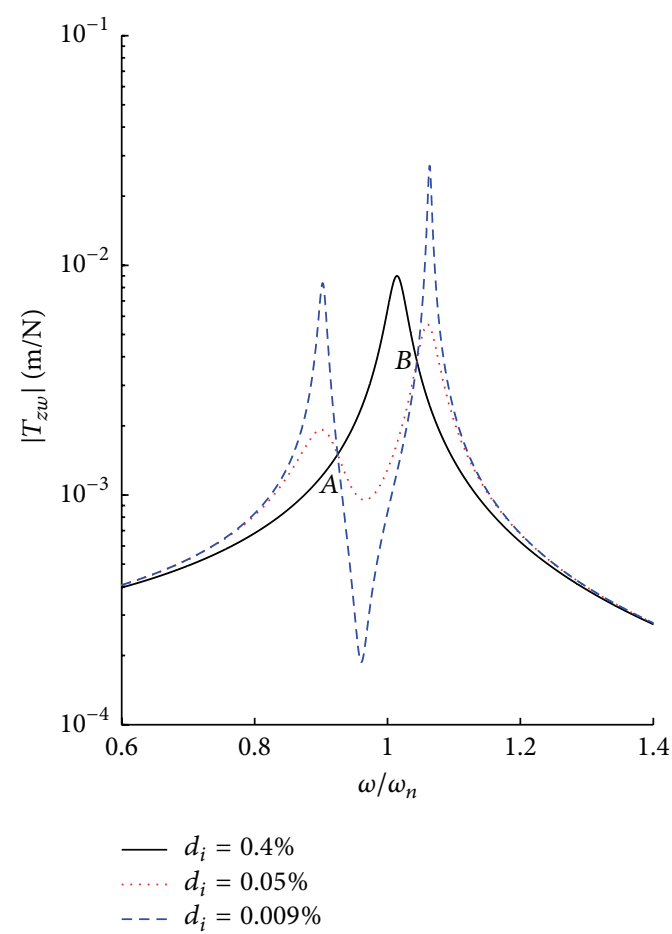

(a)

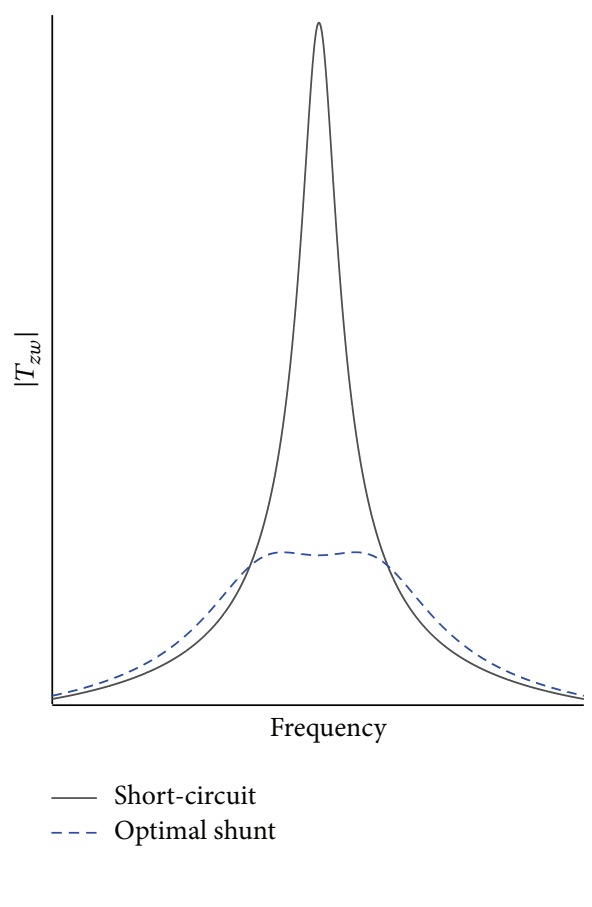

(b)

Figure 3: $\left|T_{z w}\right|$ for an undamped elastic structure (a) and trend of $\left|T_{z w}\right|$ for a generic system with the optimal value of $d_{i}$ (b).

It should be noted that the use of (13) and (15) (which are yielded considering $\xi_{n}=0$ ) in the case of damped systems introduces certain approximations. Nevertheless, these approximations can be assumed as negligible. In fact, according to [19], the maximum difference between the attenuation provided by (13) and (15) and the actual attenuation is less than $0.5 \mathrm{~dB}$ for most practical applications.

Therefore, the use of (13) and (15) can be considered reliable even with damped systems.

The behaviour of mistuned systems will be studied in the following sections. Because there are no closed formulas to describe the vibration attenuation in mistuned conditions, the maximum of $\left|T_{z w}\right|$ must be found numerically using (10). The number of variables in this equation is high: five variables, that is, $\omega_{n}, \omega_{p}, \xi_{n}, d_{i}$, and $\gamma \psi_{n}^{2}$. Hence, several simulations must be performed if a detailed description of the behaviour of different possible mistuned systems is desired (several values of $\omega_{p}, d_{i}$, and $\gamma \psi_{n}^{2}$ for each mode considered, defined by $\omega_{n}$ and $\xi_{n}$ ). Therefore, it is essential to decrease the number of variables to be considered in the simulations to reduce the effort of this numerical study.
Therefore, Section 2.1 presents a normalisation of the system model to reduce the number of variables involved in the problem.

2.1. Normalisation of the Model. First, all of the possible values of $\omega_{p}$ and $d_{i}$ can be defined as a function of the optimal ones $\omega_{p}^{\text {opt }}$ and $d_{i}^{\text {opt }}$ as follows:

$$
\begin{aligned}
d_{i} & =l d_{i}^{\mathrm{opt}} \\
\omega_{p} & =v \omega_{p}^{\mathrm{opt}},
\end{aligned}
$$

where $l$ and $v$ are the amount of mistuning on the electrical damping and eigenfrequency, respectively $(l=1$ and $v=1$ in the case of no mistuning).

Then, (10) is considered: both the numerator and the denominator are divided by $\omega_{n}^{4}$, and $s$ is expressed as $j \omega(j$ is the imaginary unit). After a few mathematical rearrangements (see Appendix A), a new expression of $T_{z w}$ in the frequency domain can be obtained. This new expression uses (13), (15), and (16) to express the electrical parameters as a function of their optimal values as follows:

$$
T_{z w}\left(x_{M}\right)=\frac{\Phi_{n}\left(x_{M}\right) \Phi_{n}\left(x_{F}\right)}{\omega_{n}^{2}} \frac{-\varphi^{2}+\sqrt{2} j \varphi k_{n} l v+v^{2}\left(1+k_{n}^{2}\right)}{\left(-\varphi^{2}+\sqrt{2} j \varphi k_{n} l v+v^{2}\left(1+k_{n}^{2}\right)\right)\left(-\varphi^{2}+2 j \xi_{n} \varphi+1\right)+j \varphi k_{n}^{2}\left(j \varphi+\sqrt{2} k_{n} l v\right)},
$$

where $\varphi=\omega / \omega_{n}$ is the nondimensional frequency. 
The advantages provided by the use of (17) will be underlined in Section 3.

\section{Attenuation Performance of the Optimally Tuned Shunt}

As previously mentioned, the performance of the shunt in terms of vibration attenuation can be expressed as the ratio between the maximum amplitude of the uncontrolled system FRF and the maximum amplitude of the controlled system FRF (i.e., $\max \left(\left|T_{z w}\right|\right)$; see (17)).

The FRF of the uncontrolled structure (i.e., with the piezoelectric patch in short-circuit) can be defined as follows [24]:

$$
G_{z w}=\frac{\Phi_{n}\left(x_{M}\right) \Phi_{n}\left(x_{F}\right)}{\left(s^{2}+2 \xi_{n} \omega_{n} s+\omega_{n}^{2}\right)} .
$$

Therefore, the attenuation performance, denoted here as att, can be expressed as follows:

$$
\begin{aligned}
\text { att } & =\frac{\max \left(\left|G_{z w}\right|\right)}{\max \left(\left|T_{z w}\right|\right)} \\
& =\frac{\left|\Phi_{n}\left(x_{M}\right) \Phi_{n}\left(x_{F}\right)\right| /\left(2 \xi_{n} \omega_{n}^{2} \sqrt{1-\xi_{n}^{2}}\right)}{\max \left(\left|T_{z w}\right|\right)},
\end{aligned}
$$

where, according to [11], $\max \left(\left|G_{z w}\right|\right)=\left|\Phi_{n}\left(x_{M}\right) \Phi_{n}\left(x_{F}\right)\right| /$ $\left(2 \xi_{n} \omega_{n}^{2} \sqrt{1-\xi_{n}^{2}}\right)$.

The analytical expression of $\max \left(\left|T_{z w}\right|\right)$ is rather complex; thus, it is convenient to define the index att $k$ instead of att for the case of perfect tuning $\left(\omega_{p}=\omega_{p}^{\mathrm{opt}}\right)$ as follows:

$$
\operatorname{att} k=\frac{\max \left(\left|G_{z w}\right|\right)}{\left|T_{z w}\right|_{\omega_{p}}}=\sqrt{\left(\frac{\max \left(\left|G_{z w}\right|\right)}{\left|T_{z w}\right|_{\omega_{p}}}\right)^{2}} .
$$

The difference between att and att $k$ is that, in the former case, the maximum amplitude of the controlled system FRF is considered $\left(\max \left(\left|T_{z w}\right|\right)\right)$, whereas, in the latter case, the value of the system response $\left|T_{z w}\right|$ at $\omega_{p}$ is considered $\left(\left|T_{z w}\right|_{\omega_{p}}\right)$. As previously mentioned, the use of att $k$ simplifies the notation and can be used to accurately approximate the value of att. In fact, in the case of perfect tuning, $\max \left(\left|T_{z w}\right|\right) \simeq\left|T_{z w}\right|_{\omega_{p}}$ because of the flat shape of $\left|T_{z w}\right|$ around $\omega_{n}\left(\omega_{p}=\omega_{p}^{\text {opt }}=\omega_{n}^{o c}\right.$ is in the frequency range where the controlled FRF has a flat shape; see Figure 3(b)) [19]. Hence, att $\approx \simeq$ att.

Based on (17), att $k$ can be expressed as follows (see Appendix B):

$$
\operatorname{att} k=\left(k_{n}+2 \sqrt{2} \xi_{n}\right) \sqrt{\frac{\left(1+k_{n}^{2}\right)}{8 \xi_{n}^{2}\left(1-\xi_{n}^{2}\right)}} .
$$

Thus, the attenuation in decibels $\left(A_{\mathrm{dB}}\right)$ can be expressed as follows:

$$
\begin{aligned}
A_{\mathrm{dB}} & =20 \log _{10} \mathrm{att} k \\
& =20 \log _{10}\left[\left(k_{n}+2 \sqrt{2} \xi_{n}\right) \sqrt{\left.\frac{\left(1+k_{n}^{2}\right)}{8 \xi_{n}^{2}\left(1-\xi_{n}^{2}\right)}\right] .}\right.
\end{aligned}
$$

Equation (22) only depends on two system parameters, $\xi_{n}$ and $k_{n}$. Therefore, the properties of tuned systems can be studied considering only these two parameters. A similar approach is used for mistuned shunt systems (see Section 4). Hence, the normalisation proposed in Section 2.1 allows the model to be simplified, thus avoiding one of the variables (i.e., now only $\xi_{n}$ and $k_{n}$ are considered, whereas it would have been necessary to consider the three parameters $\xi_{n}, \omega_{n}$, and $\gamma \psi_{n}^{2}$ without the normalisation).

It is easy to see that (22) links the achievable attenuation to the problem parameters (i.e., $k_{n}$ and $\xi_{n}$ ). Since $\xi_{n}$ is fixed, (22) allows the attenuation to be predicted as a function of the value of $k_{n}$, thus suggesting which value should be used to obtain the desired attenuation performance. In fact, it can be recalled that $k_{n}$ is a function of $\gamma \psi_{n}^{2}$ (see (12)), and it can thus be modified by changing the geometrical, mechanical, and electrical characteristics of the actuator as well as its position [19]. Furthermore, $k_{n}$ can be also modified by connecting several piezoelectric actuators in series/parallel $[25,26]$ and by using a negative capacitance $[22,27]$. Therefore, the model used here is of general validity.

Equation (22) can be rearranged as follows:

$$
\begin{aligned}
A_{\mathrm{dB}}= & 20 \log _{10}\left(k_{n}+2 \sqrt{2} \xi_{n}\right)+10 \log _{10}\left(1+k_{n}^{2}\right) \\
& -10 \log _{10}\left(8 \xi_{n}^{2}\left(1-\xi_{n}^{2}\right)\right) .
\end{aligned}
$$

Now, three different situations in terms of the $k_{n}$ value can be considered:

(1) $k_{n}$ of the same order of magnitude of $\xi_{n}$ (the maximum value of $\xi_{n}$ considered here is $1 \%$ ): this is the case of extremely stiff and damped structures and/or badly positioned actuators. In this case, (23) can be approximated as follows:

$$
\begin{aligned}
A_{\mathrm{dB}}= & 20 \log _{10}\left(k_{n}+2 \sqrt{2} \xi_{n}\right) \\
& -10 \log _{10}\left(8 \xi_{n}^{2}\left(1-\xi_{n}^{2}\right)\right) .
\end{aligned}
$$

In fact, $k_{n}$ is so low that $10 \log _{10}\left(1+k_{n}^{2}\right)$ can be approximated as $10 \log _{10}(1)=0$.

(2) $2 \sqrt{2} \xi_{n} \ll k_{n} \ll 1:$ this is the typical case [19]. It is possible to make the following simplifications: $\left(k_{n}+\right.$ $\left.2 \sqrt{2} \xi_{n}\right) \simeq k_{n}$ and $1+k_{n}^{2} \simeq 1$. Thus, for most engineering applications, (23) can be approximated as follows:

$$
\begin{aligned}
A_{\mathrm{dB}} & =20 \log _{10}\left(k_{n}\right)-10 \log _{10}\left(8 \xi_{n}^{2}\left(1-\xi_{n}^{2}\right)\right) \\
& =m_{0} \log _{10}\left(k_{n}\right)+q_{0},
\end{aligned}
$$




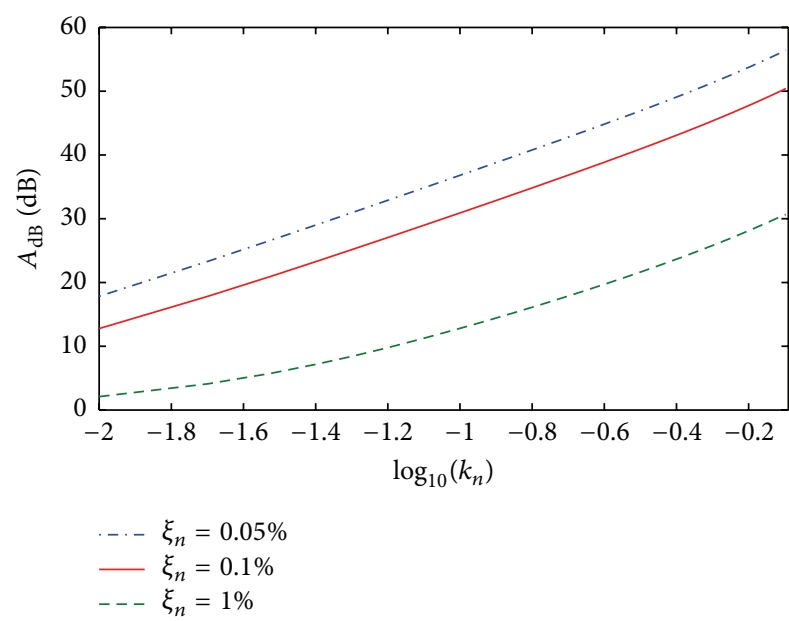

Figure 4: Relationship between $A_{\mathrm{dB}}$ and $\log _{10} k_{n}$ for different systems (i.e., different $\xi_{n}$ values).

where $m_{0}=20$ and $q_{0}=-10 \log _{10}\left(8 \xi_{n}^{2}\left(1-\xi_{n}^{2}\right)\right)$. Equation (25) demonstrates a linear relationship between $A_{\mathrm{dB}}$ and $\log _{10}\left(k_{n}\right)$.

(3) $k_{n}$ close to 1 : this is the case where extremely flexible structures and/or the addition of a negative capacitance are considered. Equation (23) can be approximated as follows:

$$
\begin{aligned}
A_{\mathrm{dB}}= & 20 \log _{10}\left(k_{n}\right)+10 \log _{10}\left(1+k_{n}^{2}\right) \\
& -10 \log _{10}\left(8 \xi_{n}^{2}\left(1-\xi_{n}^{2}\right)\right) .
\end{aligned}
$$

Equation (26) demonstrates that, in this case, the relationship between $A_{\mathrm{dB}}$ and $\log _{10}\left(k_{n}\right)$ is no longer linear. Nevertheless, in most practical applications, a linear relation can still be used. In fact, the term $10 \log _{10}\left(1+k_{n}^{2}\right)$ has a negligible contribution up to approximately $k_{n}=0.6$. Its contribution becomes more evident, albeit small, only for higher $k_{n}$ values (at $k_{n}=0.8$, its contribution is approximately $2 \mathrm{~dB}$ ). Hence, the term $10 \log _{10}\left(1+k_{n}^{2}\right)$ can be neglected, and the attenuation $A_{\mathrm{dB}}$ as a function of $\log _{10}\left(k_{n}\right)$ can be approximated by the linear relation as follows:

$$
A_{\mathrm{dB}} \simeq m_{0} \log _{10}\left(k_{n}\right)+q_{0}
$$

where $m_{0}=20$ and $q_{0}=-10 \log _{10}\left(8 \xi_{n}^{2}\left(1-\xi_{n}^{2}\right)\right)$.

Figure 4 shows the linear relationship between $A_{\mathrm{dB}}$ and $\log _{10}\left(k_{n}\right)$ for different systems selected as an example. Additionally, the figure indicates that the area in which linearity is lost (corresponding to the case in which $k_{n}$ is of the same order of magnitude of $\xi_{n}$, point 1 of the previous numbered list; see the left part of the green dashed line in the figure) corresponds to cases where $A_{\mathrm{dB}}$ is extremely low (approximately $5 \mathrm{~dB}$ or lower).
The linear relationship demonstrated thus far (see (25) and (27)) can lead to the following notations:

$$
\begin{aligned}
\log _{10} \text { att } k & \simeq \log _{10}\left(k_{n}\right)+q_{0} / 20 \Longrightarrow \\
\text { att } k & \simeq k_{n}(10)^{q_{0} / 20} \Longrightarrow \\
k_{n} & \simeq \frac{\text { att } k}{\sqrt[20]{(10)^{q_{0}}}} .
\end{aligned}
$$

The central expression of (28) indicates that if $k_{n}$ is incremented from value $f 1$ to value $f 2$ where $f 2 / f 1=g$, then the value of att $k$ increases by a factor $g$, which signifies that consistent increases in att $k$ can be achieved with moderate increases (in terms of absolute value) of $k_{n}$ when $k_{n}$ is low. Conversely, high increases in $k_{n}$ (in terms of absolute value) produce a low increment of att $k$ when $k_{n}$ is high. Hence, an asymptotic behaviour of the attenuation performance is demonstrated.

The next section considers mistuned shunt systems.

\section{Robustness of the Shunt Damping: Performance in Mistuned Conditions}

Section 1 explained that, in most cases, the shunted system operates in mistuned conditions because of the uncertainty in the estimated values of the system parameters (especially electrical quantities) or changes in either the mechanical system or the electrical network. This often leads to a control performance considerably lower than that expected; thus, a robustness analysis would be useful for understanding the behaviour of the controlled system and determining a method to limit this performance reduction. Therefore, the analysis of robustness attempts to investigate the worsening of performance due to mistuning and provides formulations for its prediction.

Based on (10), (13), and (15), the mistuning can be due to errors in the estimated values of $\xi_{n}, \omega_{n}$, and $\gamma \psi_{n}^{2}$ as well as the values of $R$ and $L$. It is easy to see that all of the different reasons for mistuning can be expressed as errors in the optimal values of $\omega_{p}$ and $d_{i}$. Therefore, in this study, the actual values of $\omega_{p}$ and $d_{i}$ are expressed as changes from their optimal values. Therefore, the mistuning can be easily considered in (17) by fixing $l$ and $v$ at values other than 1 (values lower than 1 indicate underestimation, whereas those higher than 1 indicate overestimation; see (16)). The FRF of the mistuned shunt system can thus be described by (17), and the related vibration attenuation performance is measured by the index att of (19). The attenuation in these mistuned conditions can be expressed in decibels as $A_{\mathrm{dB}}^{*}$ (see Appendix $C$ for certain clarifications for the symbols used) as follows:

$$
A_{\mathrm{dB}}^{*}=\left.20 \log _{10} \mathrm{att}\right|_{l \neq 1, v \neq 1} .
$$

The value of $A_{\mathrm{dB}}^{*}$ can be found numerically to study the attenuation in different mistuned conditions. Thus, the values of $\left|T_{z w}\right|$ as a function of frequency must be calculated for a given situation (i.e., fixing the values of $k_{n}, \xi_{n}, l$, and $v$ in (17)); 


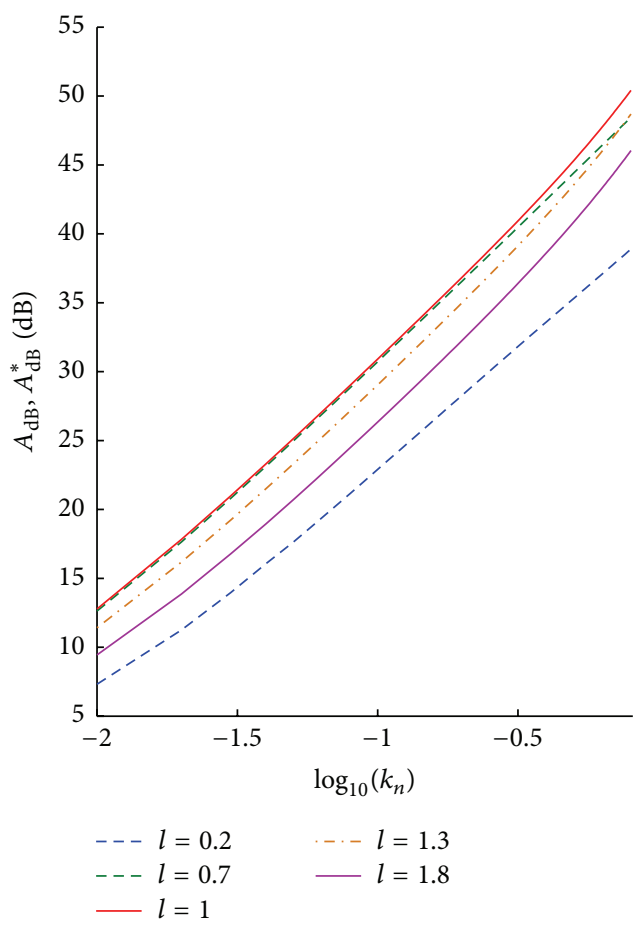

(a)

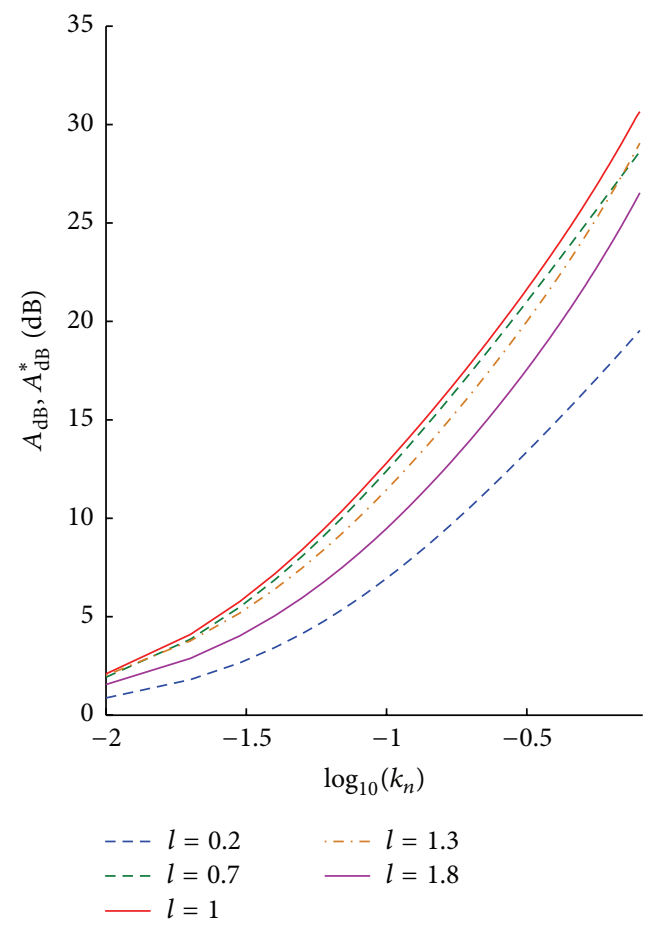

(b)

Figure 5: Relationship between $A_{\mathrm{dB}}$ or $A_{\mathrm{dB}}^{*}$ and $\log _{10} k_{n}$ for different $\xi_{n}$ values: $0.1 \%$ (a) and $1 \%$ (b).

and then the maximum of $\left|T_{z w}\right|$ can be found numerically. Lastly, att can be calculated using (19).

Nevertheless, the number of simulations needed is often high. In fact, several different values of $l$ and $v$ need to be tested to consider various different possible mistuning situations. Furthermore, numerous values of $k_{n}$ must be considered; in fact, it is useful to understand if an increased $k_{n}$ value allows the desired attenuation performance to be achieved, even in mistuned conditions. Furthermore, according to [19] and as it will be shown in Sections 4.1 and 4.3, it is often good practice to increase the initial value of the resistance with respect to its optimal value to improve the attenuation in mistuned conditions; hence, $l$ values significantly higher than 1 need to be tested, thus leading to a large number of $l$ values to be taken into account. Therefore, the number of required simulations can increase substantially. For example, when $N_{k}$ values of $k_{n}, N_{v}$ biased values of $\omega_{p}$, and $N_{l}$ biased values of $d_{i}$ have to be considered to fully study the given problem, the entire number of simulations $N_{s}$ that must be performed to evaluate the attenuation in all of the possible cases results in $N_{p} N_{v} N_{l}$ (e.g., if $N_{s}$ is equal to $10^{6}$, the amount of computational time to perform all the simulations becomes longer than 10 hours on a normal laptop).

Therefore, the goal of the next sections is to propose a model to describe the attenuation in mistuned conditions $A_{\mathrm{dB}}^{*}$. Sections 4.1 and 4.2 analyse the effects of errors on $d_{i}$ and $\omega_{p}$, respectively. Then, Section 4.3 addresses situations where both errors (i.e., on $d_{i}$ and $\omega_{p}$ ) occur together.
4.1. Mistuning on the Electrical Damping. This section only considers mistuning on $d_{i}$. Figure 5 depicts the curves relating $\log _{10} k_{n}$ and $A_{\mathrm{dB}}^{*}$ for different systems and different errors on $d_{i}$ (i.e., different $l$ values and $v=1$ ). These curves were found numerically using (29), (19), and (17) (see Section 4). In fact, a general analytical solution is not possible because the equations are of a high order, and thus the solution cannot be expressed through closed analytical formulas and must be calculated numerically case by case. It should be noted that the use of the normalised model of (17) still allows a decrease in the number of variables to be considered: four variables in the normalised model (i.e., $\xi_{n}, k_{n}$, $l$, and $v$; see (17)) versus five variables in the nonnormalised model (i.e., $\xi_{n}, \omega_{n}, \gamma \psi_{n}^{2}, l$, and $v$; see (10)).

The primary property of the plots in Figure 5 is that the main effect of mistuning is to shift the curves with respect to the case of $l=1$; however, all of these curves can still be approximated as straight lines. In fact, the lines associated with $\xi_{n}=1 \%$ lose their linear trend in correspondence of low values of $A_{\mathrm{dB}}^{*}$ (i.e., for approximately $A_{\mathrm{dB}}^{*}<4 \mathrm{~dB}$ ); nevertheless, these curves can still be considered piecewise linear. In fact, if an interval for $k_{n}$ equal to one order of magnitude is considered (it is hard to change $k_{n}$ for one order of magnitude [25] or more, even using negative capacitances [27]), the curves can be well approximated as lines.

The lines in Figure 5 prove that the effects of the change in the intercept are significantly higher than the effects of the change in the angular coefficient (i.e., the lines primarily shift 
due to a nonunitary value of $l$, parallel lines). In other words, the sensitivity of the attenuation performance on the value of the coupling coefficient tends to be independent of the level of mistuning. Hence, for a given system, the improvement in the attenuation achieved by increasing the value of the coupling coefficient is the same whether the shunt is tuned or not.

The relationship between $\log _{10} k_{n}$ and $A_{\mathrm{dB}}^{*}$ for a given system can be expressed as follows:

$$
A_{\mathrm{dB}}^{*} \simeq q_{l}^{*}(l)+m_{l}^{*}(l) \log _{10}\left(k_{n}\right)=\widetilde{A}_{\mathrm{dB}}^{*},
$$

where $\widetilde{A}_{\mathrm{dB}}^{*}$ is the estimate of $A_{\mathrm{dB}}^{*}$ and $q_{l}^{*}$ and $m_{l}^{*}$ are the intercept and the angular coefficient of the line, respectively, which are both a function of $l$, as evidenced in (30) (see also Appendix $\mathrm{C}$ for certain clarifications of the symbols used). It should be noted that $m_{l}^{*}$ is indicated as dependent on $l$, even if this dependency is slight (see above), for the sake of completeness.

If the trend of $A_{\mathrm{dB}}^{*}$ as a function of $l$ is depicted for different values of $k_{n}$, a few further interesting facts can be noted (see Figure 6). All of the curves of this new figure demonstrate nearly the same features: the attenuation loss is limited for $l>1$; furthermore, in this range of $l$, the rate of the loss is nearly constant. Conversely, for $l<1$, the rate becomes increasingly larger by decreasing the value $l$. It is possible to approximatively state that, for $l<0.5$, the rate of the attenuation loss increases. This result is a first sign of the benefits provided by the use of overestimated electrical damping $d_{i}$ values (and thus overestimated $R$ values). In fact, even if an overestimated $d_{i}$ value causes a worsening in the attenuation performance if compared to the tuned situation, this worsening is not that high (see Figure 6); overall, if a mistuning occurs in situations where the starting $d_{i}$ value is overestimated deliberately, the attenuation loss due to the mistuning is low. The use of initially overestimated $d_{i}$ values will be considered again in Section 4.3.

A further interesting point is that the trend of $A_{\mathrm{dB}}^{*}$ as a function of $l$ can be modelled as the combination of two fourth-order polynomials, one for $l<1$ and another for $l>1$, regardless of the system considered (see Figure 6).

The calculation for each of these fourth-order polynomials requires the knowledge of $A_{\mathrm{dB}}^{*}$ for five values of $l$. Therefore, for the given values of $\xi_{n}$ and $k_{n}$, it is sufficient to calculate ten points $\left(l, A_{\mathrm{dB}}^{*}\right)$ using (29), (19), and (17) (i.e., five for $l<1$ and five for $l>1)$ to determine the trend of $A_{\mathrm{dB}}^{*}$ for an extended range of $l$ values (e.g., $l=0.01 \div 2$, as indicated in Figure 6).

Based on (30) and Figure 6, the following procedure can be applied when the behaviour of a mistuned shunt system in a range of $k_{n}$ values between $k_{A}$ and $k_{B}$ is studied.

(i) Calculate five pairs ( $\left.l, A_{\mathrm{dB}}^{*}\right)$ using (29), (19), and (17) for $l<1$ and $k_{n}=k_{A}$ and determine the interpolating polynomial. Then, repeat the same procedure for $l>$ 1. It is now possible to know the value of $A_{\mathrm{dB}}^{*}$ for any value of $l$ at $k_{n}=k_{A}$.

(ii) Calculate five pairs $\left(l, A_{\mathrm{dB}}^{*}\right)$ using (29), (19), and (17) for $l<1$ and $k_{n}=k_{B}$ and determine the interpolating polynomial. Then, repeat the same procedure for
TABLE 1: Bounds for the Monte Carlo simulations for $l \neq 1$ and $v=1$.

\begin{tabular}{ccccc}
\hline & $\xi_{n}$ & $k_{A}$ & $k_{n}$ & $l_{x}$ \\
\hline $\operatorname{Min}$ & $0.05 \%$ & 0.001 & $k_{A}$ & 0.5 \\
$\operatorname{Max}$ & $1 \%$ & 0.35 & $k_{B}$ & 1.5 \\
\hline
\end{tabular}

$l>1$. It is now possible to know the value of $A_{\mathrm{dB}}^{*}$ for any value of $l$ at $k_{n}=k_{B}$.

(iii) The value of $\widetilde{A}_{\mathrm{dB}}^{*}$ for a generic value $k_{n}$ between $k_{A}$ and $k_{B}$ and a generic value $l=l_{x}$ can be computed using (30), where

$$
\begin{aligned}
& m_{l}^{*}=\frac{\left.A_{\mathrm{dB}}^{*}\right|_{l=l_{x}, k_{n}=k_{B}}-\left.A_{\mathrm{dB}}^{*}\right|_{l=l_{x}, k_{n}=k_{A}}}{\log _{10} k_{B}-\log _{10} k_{A}} \\
& q_{l}^{*}=\left.A_{\mathrm{dB}}^{*}\right|_{l=l_{x}, k_{n}=k_{B}}-m_{l}^{*} \log _{10} k_{B} .
\end{aligned}
$$

Therefore, it is possible to estimate the attenuation for any value of $l$ and $k_{n}$ (between $k_{A}$ and $k_{B}$ ) with only twenty simulations.

The accuracy of this procedure was verified using a Monte Carlo test with more than $10^{5}$ simulations comparing the attenuation values $\widetilde{A}_{\mathrm{dB}}^{*}$ achieved using this procedure and the $A_{\mathrm{dB}}^{*}$ values obtained using (29), (19), and (17). The difference $\Delta$ is defined as $\widetilde{A}_{\mathrm{dB}}^{*}-A_{\mathrm{dB}}^{*}$. For each simulation, the values of $\xi_{n}, k_{A}, k_{n}$, and $l_{x}$ were extracted from uniform distributions. The bounds of the distributions are presented in Table 1 and were chosen in order to take into account the most part of the practical applications. Table 2 lists the results, hence proving the reliability of the proposed procedure. It should be noted that $k_{B}=\sqrt{3} k_{A}$ was used in the simulations. This corresponds to a change of $\gamma \psi_{n}^{2}$ within an interval equal to three times the starting value, which is quite a broad interval. If a wider interval of $k_{n}$ must be considered and the same accuracy is desired, it is possible to analyse the system in two different ranges. For example, if $k_{B}=3 k_{A}$, the entire range can be split as follows: $k_{A} \div k_{h}$ and $k_{h} \div k_{B}$ with $k_{h}=\sqrt{3} k_{A}$. This requires using thirty simulations instead of twenty to describe the behaviour of the mistuned shunt system.

4.2. Mistuning on the Electrical Eigenfrequency. Figure 7 illustrates the same information as Figure 5 but for mistuning on $\omega_{p}$ (i.e., $v \neq 1$ and $l=1$ ). It should be noted that the resulting curves tend to increase their curvature. Nevertheless, the linearity is lost only when $A_{\mathrm{dB}}^{*}$ becomes lower than approximately 4 or $5 \mathrm{~dB}$. However, the curves can be still piecewise approximated as lines with enough accuracy for wide ranges of $\log _{10}\left(k_{n}\right)$. The effect of a nonunitary value of $v$ is to highly increase the angular coefficient of the lines. Consequently, increasing the value of $k_{n}$ is even more effective in enhancing the attenuation in the case of mistuning on $\omega_{p}$ than in the case of tuned systems.

The curves of Figure 7 can be expressed as follows:

$$
A_{\mathrm{dB}}^{*} \simeq q_{v}^{*}(v)+m_{v}^{*}(v) \log _{10}\left(k_{n}\right)=\widetilde{A}_{\mathrm{dB}}^{*},
$$

where $\widetilde{A}_{\mathrm{dB}}^{*}$ is again the estimate of $A_{\mathrm{dB}}^{*}$ and $q_{v}^{*}$ and $m_{v}^{*}$ are the intercept and the angular coefficient of the lines, respectively, 


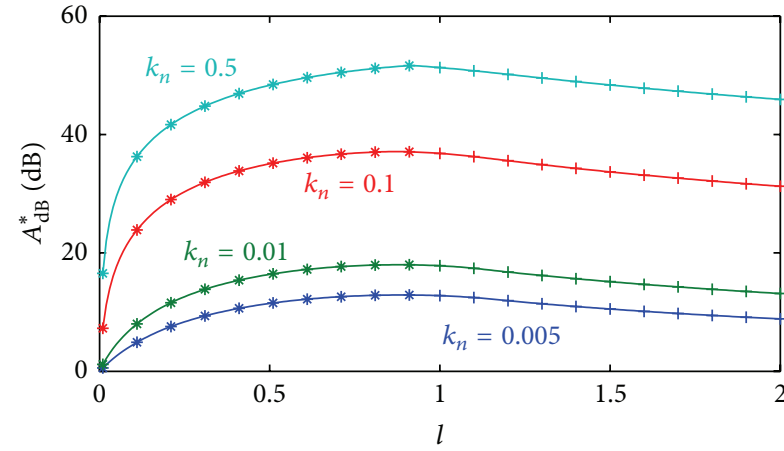

(a)

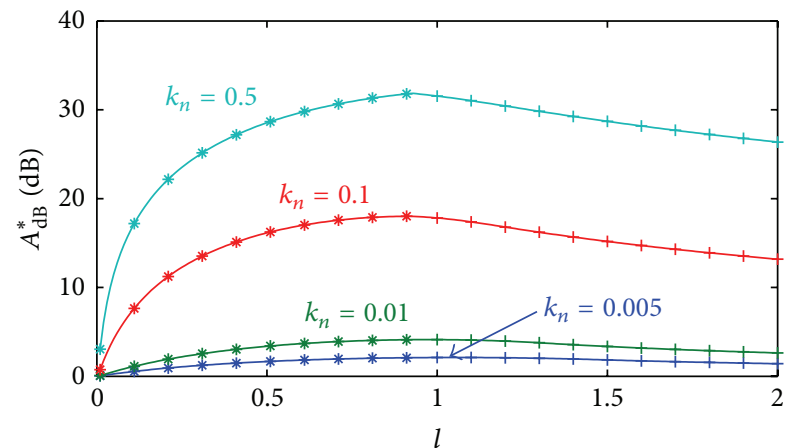

(c)

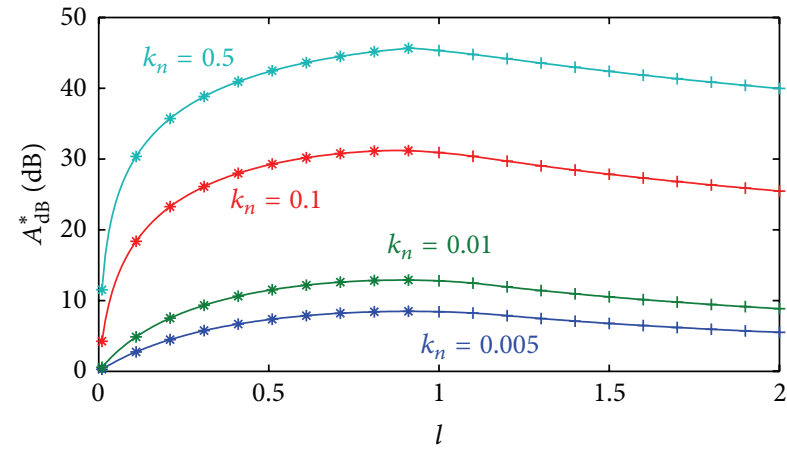

(b)

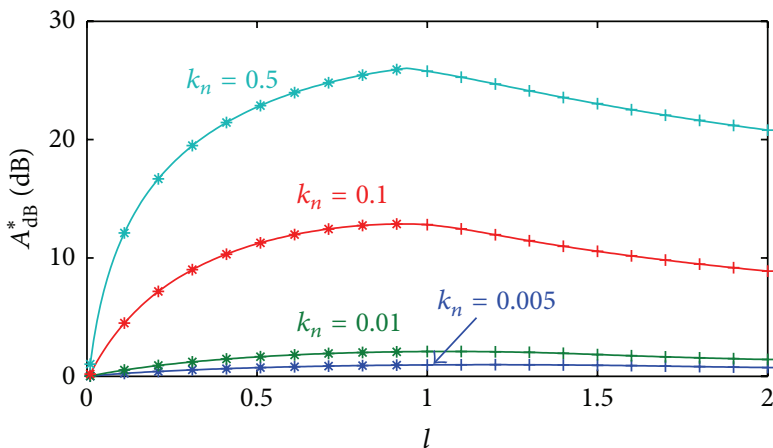

(d)

FiguRE 6: Relationship between $A_{\mathrm{dB}}^{*}$ and $l$ for different $k_{n}$ values and different $\xi_{n}$ values: $0.05 \%$ (a), $0.1 \%$ (b), $0.5 \%$ (c), and $1 \%$ (d). The + are the points related to the case of $l>1$ calculated using (29), (19), and (17), which are then interpolated by fourth-order polynomials (solid curves). The $*$ are the points related to the case of $l<1$ calculated using (29), (19), and (17), which are then interpolated by fourth-order polynomials (solid curves).

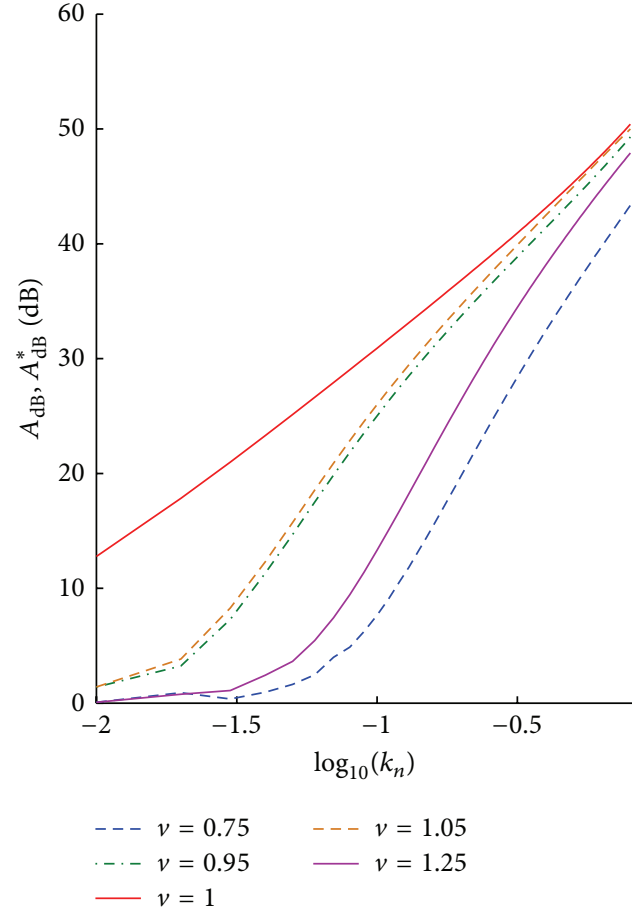

(a)

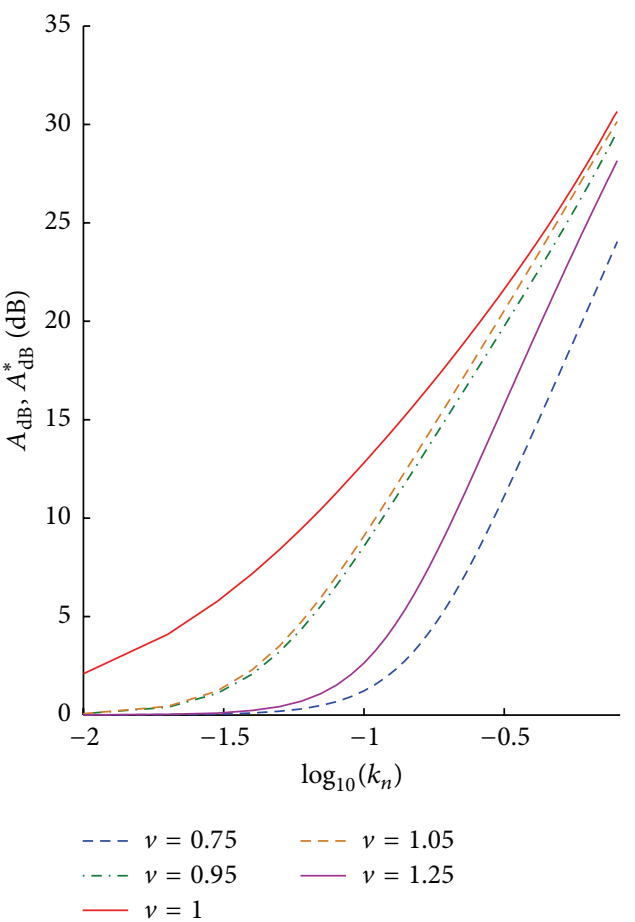

(b)

FIGURE 7: Relationship between $A_{\mathrm{dB}}$ or $A_{\mathrm{dB}}^{*}$ and $\log _{10} k_{n}$ for different $\xi_{n}$ values: $0.1 \%$ (a) and $1 \%$ (b). 


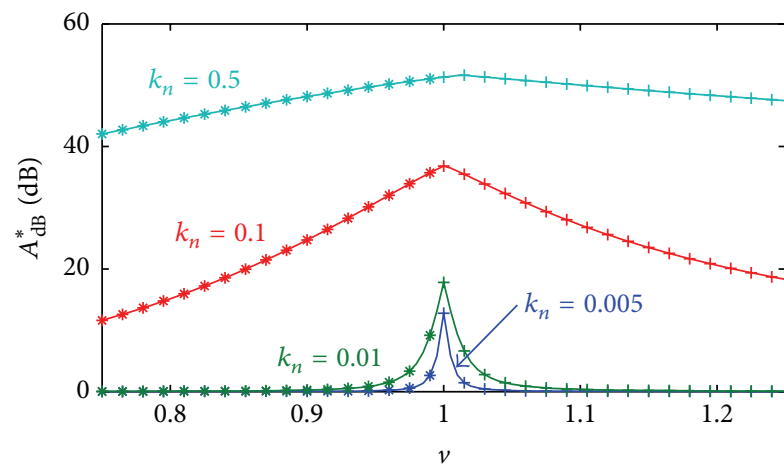

(a)

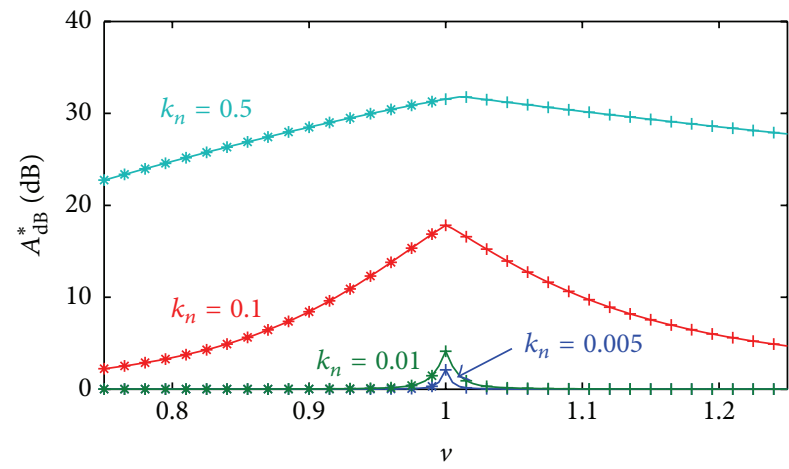

(c)

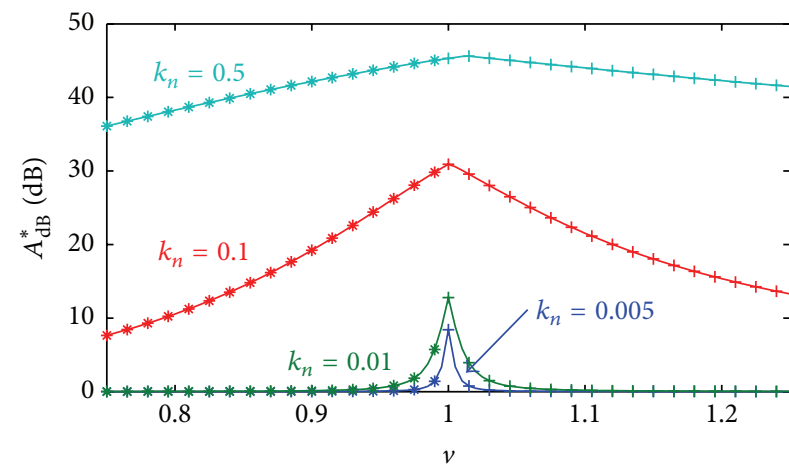

(b)

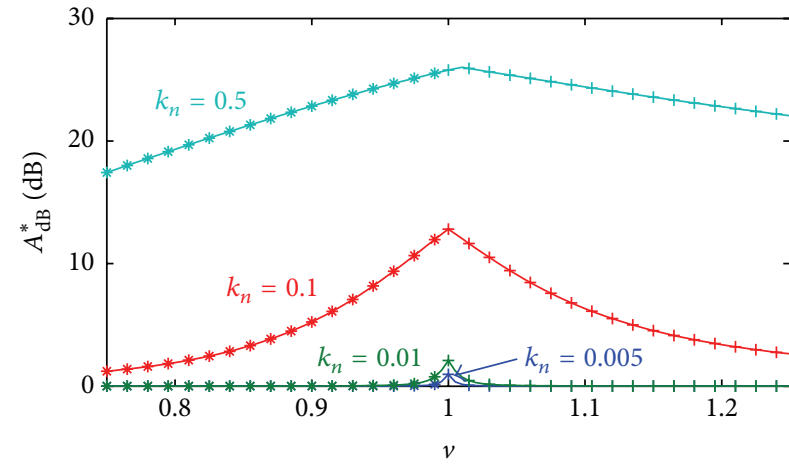

(d)

Figure 8: Relationship between $A_{\mathrm{dB}}^{*}$ and $v$ for different $k_{n}$ values and different $\xi_{n}$ values: $0.05 \%$ (a), $0.1 \%$ (b), $0.5 \%$ (c), and $1 \%$ (d). The + are the points related to the case of $v>1$ calculated using (29), (19), and (17), which are then interpolated by fourth-order polynomials (solid curves). The $*$ are the points related to the case of $v<1$ calculated again using (29), (19), and (17), which are then interpolated by fourth-order polynomials (solid curves).

TABLE 2: Results of the Monte Carlo simulations for the case $l \neq 1$ and $v=1$.

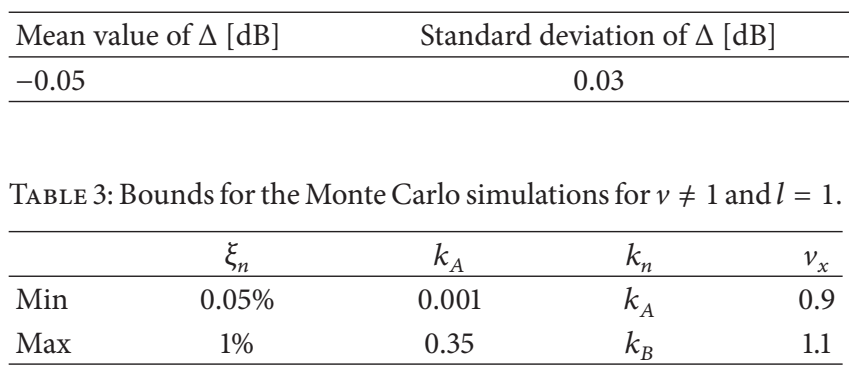

which are both functions of $v$ (see Appendix C for certain clarifications of the symbols used).

If the trend of $A_{\mathrm{dB}}^{*}$ is shown as a function of $v$ for different values of $k_{n}$, a few further interesting facts can be noted (see Figure 8). The same percentage value of mistuning leads to a different decrease in the performance if it is related to an overestimation or underestimation of the optimal value of the electrical eigenfrequency. In fact, values of $v$ lower than 1 cause higher losses in the attenuation than values greater than 1 (e.g., compare the curves at $v=0.75$ and $v=1.25$ ).

A further interesting point is that the trend of $A_{\mathrm{dB}}^{*}$ as a function of $v$ can be modelled as a fourth-order polynomial for both $v<1$ and $v>1$, regardless of the system considered (see Figure 8). Therefore, if the study of the behaviour of a

mistuned shunt system in a range of $k_{n}$ values between $k_{A}$ and $k_{B}$ is considered, the same procedure discussed in Section 4.1 (see the list in Section 4.1) can be applied, and it is possible to estimate the attenuation for any value of $v$ and $k_{n}$ (between $k_{A}$ and $k_{B}$ ) with only twenty simulations. Indeed, it is possible to write

$$
\begin{gathered}
m_{v}^{*}=\frac{\left.A_{\mathrm{dB}}^{*}\right|_{v=v_{x}, k_{n}=k_{B}}-\left.A_{\mathrm{dB}}^{*}\right|_{v=v_{x}, k_{n}=k_{A}}}{\log _{10} k_{B}-\log _{10} k_{A}} \\
q_{v}^{*}=\left.A_{\mathrm{dB}}^{*}\right|_{v=v_{x}, k_{n}=k_{B}}-m_{v}^{*} \log _{10} k_{B} .
\end{gathered}
$$

Again, a Monte Carlo test was performed with more than $10^{5}$ simulations comparing the attenuation values $\widetilde{A}_{\mathrm{dB}}^{*}$ achieved using this procedure and the $A_{\mathrm{dB}}^{*}$ values obtained using (29), (19), and (17). For each simulation, the values of $\xi_{n}, k_{A}, k_{n}$, and $v_{x}$ were extracted from uniform distributions (see Table 3 for the bounds of the distributions, which were chosen in order to take into account the most part of the practical applications), and $k_{B}$ was fixed to $\sqrt{3} k_{A}$, as performed in Section 4.1. Table 4 lists the results, thus proving the reliability of the proposed 
TABLE 4: Results of the Monte Carlo simulations for $v \neq 1$ and $l=1$.

\begin{tabular}{lccc}
\hline Mean value of $\Delta[\mathrm{dB}]$ & Standard deviation of $\Delta[\mathrm{dB}]$ & Minimum value of $\Delta[\mathrm{dB}]$ & Maximum value of $\Delta[\mathrm{dB}]$ \\
\hline-0.02 & 0.07 & -0.50 & 0.19 \\
\hline
\end{tabular}

procedure. It should be noted that the range of $v_{x}$ is narrower than that used in Section 4.1 for $l_{x}$. The reason is that the optimal value of $d_{i}$ depends on more variables than the optimal value of $\omega_{p}$, thus leading to more uncertainty (see (15) and (13)).

\subsection{Mistuning on Both the Electrical Eigenfrequency and the} Damping Ratio. Sections 4.1 and 4.2 have treated cases in which the mistuning is related to either $d_{i}$ or $\omega_{p}$. Nevertheless, in actual applications, both of the mistuning effects are expected to appear together.

In these general mistuned situations (i.e., $l \neq 1$ and $v \neq 1$ ), the performance of the shunt system demonstrates a different behaviour for values of $l$ higher or lower than 1 . This result can be clearly evidenced using the double-logarithmic representation already utilised in Figures 5 and 7. In fact, Figure 9 depicts the different behaviour for certain systems selected as examples:

(i) For systems where $l>1$, the loss of attenuation is essentially due to the mistuning causing the highest loss (see the subplots on the right side).

(ii) For systems where $l<1$, the loss of attenuation can be derived as the sum of the losses caused by both the mistuning types (see the subplots on the left side).

Now, for a given system (i.e., fixed values of $\xi_{n}$ and $k_{n}$ ) and fixed values of $l$ and $v$ (named $l_{x}$ and $v_{x}$ ), the following indexes can be defined:

$$
\begin{aligned}
& y_{t}=A_{\mathrm{dB}}-\left.A_{\mathrm{dB}}^{*}\right|_{l=l_{x}, v=v_{x}} \\
& y_{o}=A_{\mathrm{dB}}-\left.A_{\mathrm{dB}}^{*}\right|_{l=1, v=v_{x}} \\
& y_{d}=A_{\mathrm{dB}}-\left.A_{\mathrm{dB}}^{*}\right|_{l=l_{x}, v=1},
\end{aligned}
$$

where $y_{t}$ expresses the loss of attenuation when a mistuning occurs on the values of both $\omega_{p}$ and $d_{i} ; y_{o}$ expresses the loss of attenuation when a mistuning occurs only on the value of $\omega_{p}$; and $y_{d}$ expresses the loss of attenuation when a mistuning occurs only on the value of $d_{i}$.
Based on the abovementioned considerations related to Figure 9 (see the list above in this section), $\tilde{y}_{t}$ (the estimate of $\left.y_{t}\right)$ can be calculated as follows:

$$
\tilde{y}_{t}= \begin{cases}\max \left(y_{o}, y_{d}\right), & \forall l_{x}>1 \\ y_{o}+y_{d}, & \forall l_{x}<1\end{cases}
$$

According to (35), $\widetilde{A}_{\mathrm{dB}}^{*}$ (i.e., the estimate of $A_{\mathrm{dB}}^{*}$ ) can be defined as follows:

$$
\begin{aligned}
& \left.\widetilde{A}_{\mathrm{dB}}^{*}\right|_{l=l_{x}, v=v_{x}} \\
& \quad= \begin{cases}\min \left(\left.A_{\mathrm{dB}}^{*}\right|_{l=1, v=v_{x}},\left.A_{\mathrm{dB}}^{*}\right|_{l=l_{x}, v=1}\right), & \forall l_{x}>1 \\
\left.A_{\mathrm{dB}}^{*}\right|_{l=1, v=v_{x}}+\left.A_{\mathrm{dB}}^{*}\right|_{l=l_{x}, v=1}-A_{\mathrm{dB}}, & \forall l_{x}<1 .\end{cases}
\end{aligned}
$$

$\left.A_{\mathrm{dB}}^{*}\right|_{l=1, v=v_{x}}$ and $\left.A_{\mathrm{dB}}^{*}\right|_{l=l_{x}, v=1}$ can be estimated using (32) and (30), respectively. $A_{\mathrm{dB}}$ is given in (22). Hence, (36) allows the attenuation to be estimated for any values of $l, v$, and $k_{n}$ (between $k_{A}$ and $k_{B}$ ) using only forty-two simulations based on (17), (19), and (29). In fact, (36) allows the behaviour of the mistuned shunt systems to be analysed with a bias on both $\omega_{p}$ and $d_{i}$ by considering the mistuning on $\omega_{p}$ and $d_{i}$ separately. Twenty simulations are needed to study the behaviour of the system with $l \neq 1$ and $v=1$ (see Section 4.1), twenty for the case $l=1$ and $v \neq 1$ (see Section 4.2), and two for the case $l=1$ and $v=1$ (i.e., one with $k_{n}=k_{A}$ and the other with $k_{n}=k_{B}$ ); furthermore, the $A_{\mathrm{dB}}$ (i.e., the case with $l=1$ and $v=1$ ) values for $k_{n}=k_{A}$ and $k_{n}=k_{B}$ can also be calculated using (22). In the case of $N_{s}$ (total number of cases to be considered) equal to $10^{6}$, the amount of time required to perform all the simulations decreases from more than 10 hours (see the end of Section 4) to a few minutes or less (approximately $30 \mathrm{~s}$ ). Hence, the study of the behaviour of the system in mistuned conditions becomes very fast, thus allowing to quickly analyse the effects of different $R$ values on the attenuation performance and to choose the best one for the given application.

Therefore, by rearranging (36) using (30) and (32), the final form of the approximated model able to describe the behaviour of the mistuned shunt systems can be achieved as follows:

$$
\left.\widetilde{A}_{\mathrm{dB}}^{*}\right|_{l=l_{x}, v=v_{x}, k_{n}}= \begin{cases}\min \left(q_{v}^{*}\left(v_{x}\right)+m_{v}^{*}\left(v_{x}\right) \log _{10}\left(k_{n}\right), q_{l}^{*}\left(l_{x}\right)+m_{l}^{*}\left(l_{x}\right) \log _{10}\left(k_{n}\right)\right), & \forall l_{x}>1 \\ q_{v}^{*}\left(v_{x}\right)+m_{v}^{*}\left(v_{x}\right) \log _{10}\left(k_{n}\right)+q_{l}^{*}\left(l_{x}\right)+m_{l}^{*}\left(l_{x}\right) \log _{10}\left(k_{n}\right)-\left.A_{\mathrm{dB}}\right|_{k_{n}}, & \forall l_{x}<1\end{cases}
$$

where $m_{l}^{*}, q_{l}^{*}, m_{v}^{*}$, and $q_{v}^{*}$ are defined in (31) and (33).

The accuracy of this model was tested again using a Monte Carlo simulation with more than $10^{6}$ cases, thus comparing the attenuation values $\widetilde{A}_{\mathrm{dB}}^{*}$ achieved using this procedure and the $A_{\mathrm{dB}}^{*}$ values obtained using (29), (19), and (17). For each simulation, the values of $\xi_{n}, k_{A}, k_{n}, v_{x}$, and $l_{x}$ were extracted 


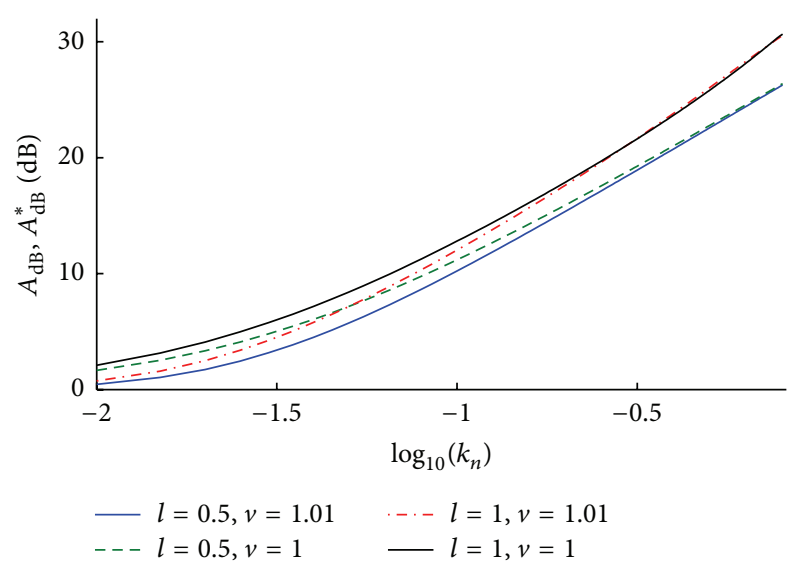

(a)

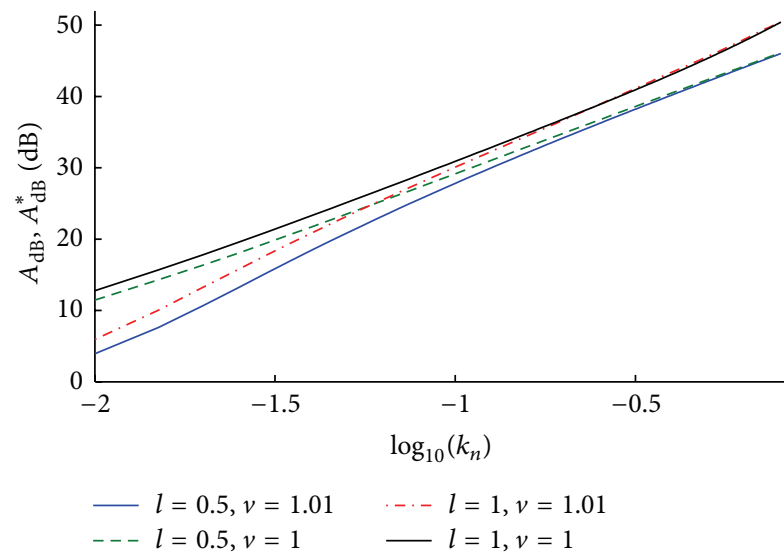

(c)

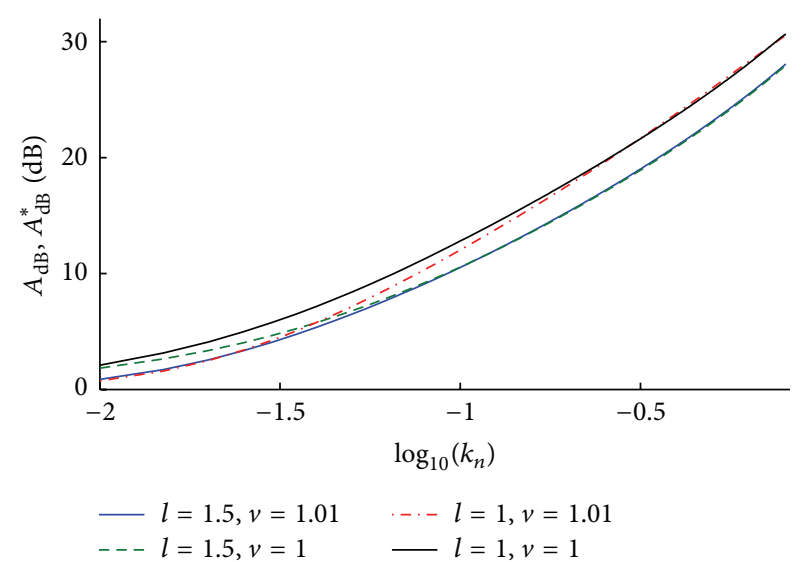

(b)

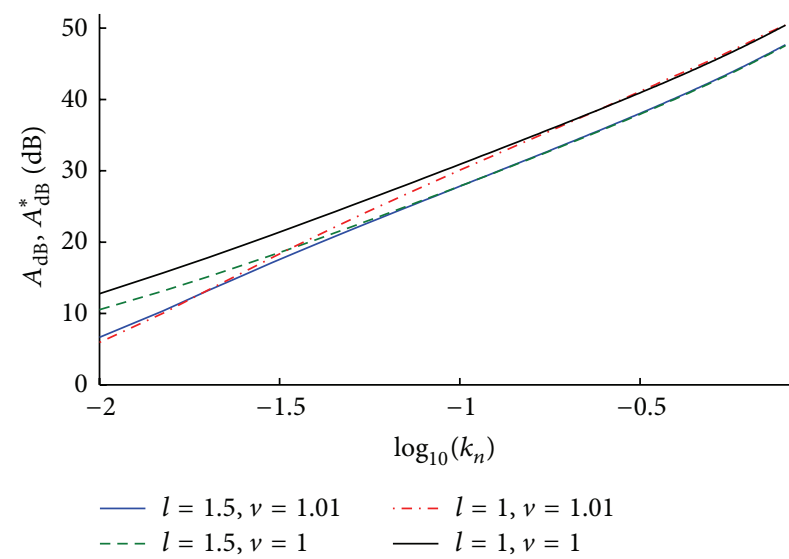

(d)

Figure 9: Relationship between $A_{\mathrm{dB}}$ or $A_{\mathrm{dB}}^{*}$ and $\log _{10} k_{n}$ for different $\xi_{n}$ values, $l$, and $v: \xi_{n}=1 \%, l=0.5$, and $v=1.01$ (a), $\xi_{n}=1 \%, l=1.5$, and $v=1.01(\mathrm{~b}), \xi_{n}=0.1 \%, l=0.5$, and $v=1.01(\mathrm{c})$, and $\xi_{n}=0.1 \%, l=1.5$, and $v=1.01(\mathrm{~d})$.

from uniform distributions (see Table $5 ; k_{B}=\sqrt{3} k_{A}$ ). Table 6 presents the results (which have a Gaussian distribution), thus proving the reliability of the proposed procedure.

Certain benefits provided by the use of initially overestimated $d_{i}$ values have already been discussed for the cases of bias just on $d_{i}$ in Section 4.1. Here, the discussion can be extended to the more general case of mistuning on both $d_{i}$ and $\omega_{p}$ (which is the typical situation). Also in this case the use of an initially overestimated $d_{i}$ value allows the loss of attenuation to decrease. In fact, when $d_{i}$ is overestimated, only one bias has significant effects, whereas the other does not have much influence (see above in this section and Figure 9). Conversely, when the $d_{i}$ value is lower than its optimal value, the attenuation loss due to mistuning is more severe. Therefore, this property of the mistuned systems along with those already shown in Section 4.1 highlights that the use of initially overestimated $d_{i}$ values (and thus initially overestimated $R$ values) allows the robustness to increase, thus lowering the loss of attenuation due to mistuning, which is typically experienced starting from the optimal $d_{i}$ value. Furthermore, this can allow the analysis of the mistuned system to become faster because the study of its behaviour can focus on values of $l$ higher than 1 (because an initially overestimated $d_{i}$ value is used on purpose) and possibly slightly lower than 1 (e.g., greater than 0.5 ). Clearly, these are just guidelines because each practical case could require a different solution. Nevertheless, the points demonstrated thus far clearly indicate how robustness can be typically increased and how the proposed model can help in the tuning process.

The model presented so far has been validated by experimental tests shown in the next section.

\section{Experimental Tests}

This section describes the experimental tests performed to validate the results shown in the previous sections. Two test structures have been used to investigate different values of the $\xi_{n}$ and $k_{n}$ parameters and different values of vibration attenuation. The first structure is an aluminium plate (in freefree condition by suspension) with the shunted piezobender bonded at about its centre (see Figure 10(a)). A bidimensional structure was used because it is a more complex test case when compared to monodimensional structures often used in other studies. The plate length is $600 \mathrm{~mm}$, the width is $400 \mathrm{~mm}$, and the thickness is $8 \mathrm{~mm}$ (this set-up is the same 


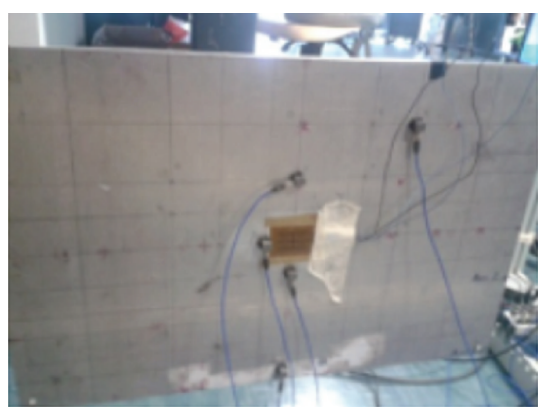

(a)

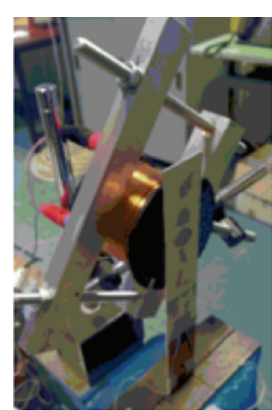

(b)
FIgURE 10: Experimental set-ups: plate (a) and beam (b).

TABLE 5: Bounds for the Monte Carlo simulations for $v \neq 1$ and $l \neq 1$.

\begin{tabular}{cccccc}
\hline & $\xi_{n}$ & $k_{A}$ & $k_{n}$ & $l_{x}$ & $v_{x}$ \\
\hline Min & $0.05 \%$ & 0.001 & $k_{A}$ & 0.5 & 0.9 \\
Max & $1 \%$ & 0.35 & $k_{B}$ & 1.5 & 1.1 \\
\hline
\end{tabular}

as that used in the experiments of [19]). The capacitance $C_{p}$ is $0.02 \mu \mathrm{F}$. Several modes were taken into account in the tests. The one (among others tested) considered here as an example has the following modal parameters (identified by experimental modal analysis [28]): $\omega_{n}=530.67 \cdot 2 \pi \mathrm{rad} / \mathrm{s}$, $\xi_{n}=0.22 \%, k_{n}=0.0081$, and $\gamma \psi_{n}^{2}=725 \mathrm{rad}^{2} / \mathrm{s}^{2}$. Actually, the value of $k_{n}$ was estimated by testing the system in both shortand open-circuit conditions (see (12)).

These values of $\gamma \psi_{n}^{2}$ and $k_{n}$ were achieved using a negative capacitance [27], which allowed their initial low values to increase. Furthermore, another value of $k_{n}$ (i.e., 0.0240) was tested by further boosting the negative capacitance performance. The disturbance to the structure was provided by a dynamometric impact hammer, and the response was measured using a piezoelectric accelerometer.

The second structure is an aluminium cantilever beam (159 mm length, $25 \mathrm{~mm}$ width, and $1 \mathrm{~mm}$ thickness) with a piezoelectric patch bonded corresponding to the clamped end (see Figure 10(b)). Its capacitance $C_{p}$ is $31 \mathrm{nF}$. Again, several modes were considered during the tests; here, the results related to the first mode are presented for the sake of conciseness. It has the following modal parameters, again identified using an experimental modal analysis: $\omega_{n}=32.61$. $2 \pi \mathrm{rad} / \mathrm{s}, \xi_{n}=0.40 \%, k_{n}=0.2002$, and $\gamma \psi_{n}^{2}=944.6 \mathrm{rad}^{2} / \mathrm{s}^{2}$. Furthermore, other tests were performed by increasing the values of $k_{n}$ up to 0.5108 using a negative capacitance.

Because this second test structure was extremely light, noncontact methods were used to provide excitation and to measure the response. Indeed, an electromagnetic device was used to excite the structure [29], and the response was measured using a laser velocimeter focused on the beam tip. The tests were performed by exciting the beam with a random signal [30] up to $1.6 \mathrm{kHz}$.

The tests were performed using synthetic impedance based on operational amplifiers [11, 31, 32] to build the inductor. Actually, certain tests on the plate were performed using an additional method: the entire shunt impedance was simulated using a high-speed Field Programmable Gate Array (FPGA) device (in this second case, a colocated piezoelectric patch was used to provide the input voltage to the simulated shunt impedance). The use of the FPGA device allowed for the full control of the parameters of the electric shunt impedance. Nevertheless, the two techniques led to similar results; therefore, those achieved using the synthetic impedance are presented here, since this technique introduces the highest level of uncertainty between the two. Therefore, the authors believed it to be the most representative to demonstrate the model effectiveness.

First, the reliability of the model, represented by (10) and (17), was verified. Figure 11 depicts the FRFs for the mode at approximately $530 \mathrm{~Hz}$ of the plate, achieved with different configurations of the shunt (i.e., using different $l$ and $v$ values). The numerical FRFs match the experimental curves, thus confirming the accuracy of the numerical model. The curves are not plotted on the same graph for the sake of clarity in the figure. Nonetheless, Figure 12 depicts a few of the experimental and numerical FRFs of Figure 11 on the same plot for an easy comparison. Then, the reliability of the proposed approximated model (see (37)) for predicting the attenuation in mistuned conditions was tested. Tables 7 and 8 list the comparisons between experimental attenuations, numerical attenuations calculated using the theoretical model of (17), (19), and (29), and attenuations estimated using the proposed approximated model of (37) for the plate and the beam. To build the approximated model of (37), the values of $k_{A}$ and $k_{B}$ must be fixed. Three different situations were tested: one where $k_{A}$ was close to $k_{n}\left(k_{n}=1.1 k_{A}\right.$, named case $1)$, one where $k_{B}$ was close to $k_{n}\left(k_{n}=1.63 k_{A}\right.$, named case 2$)$, and a further one where $k_{n}$ was nearly halfway $\left(k_{n}=1.37 k_{A}\right.$, named case 3). In all of the three cases, $k_{B}$ was fixed to $\sqrt{3} k_{A}$. The experimental attenuations are defined in the tables as EA, whereas the numerical ones (see (17), (19), and (29)) are defined as NA for the sake of conciseness. Moreover, the attenuation provided by the model of (37) in cases 1,2 , and 3 is named MA1, MA2, and MA3, respectively. The match among all of the results is good. The results related to the MA1, MA2, and MA3 cases are always close to each other, and the maximum difference if compared to the NA results is on the order of $0.5 \mathrm{~dB}$. Because the EA results differ from the NA results at a maximum of $1.2 \mathrm{~dB}$, the proposed model of (37) is considered to be validated.

\section{Conclusion}

This paper addresses monomodal vibration attenuation using piezoelectric transducers shunted to impedances consisting of an inductance and a resistance in series. Although this method works well when the tuning between the mechanical system and the electrical network is properly realised, this control technique is not adaptive, and its performances thus decrease as soon as a mistuning occurs.

The paper analyses the behaviour of mistuned electromechanical systems, demonstrating that a linear relationship between the attenuation and the logarithm of the effective 


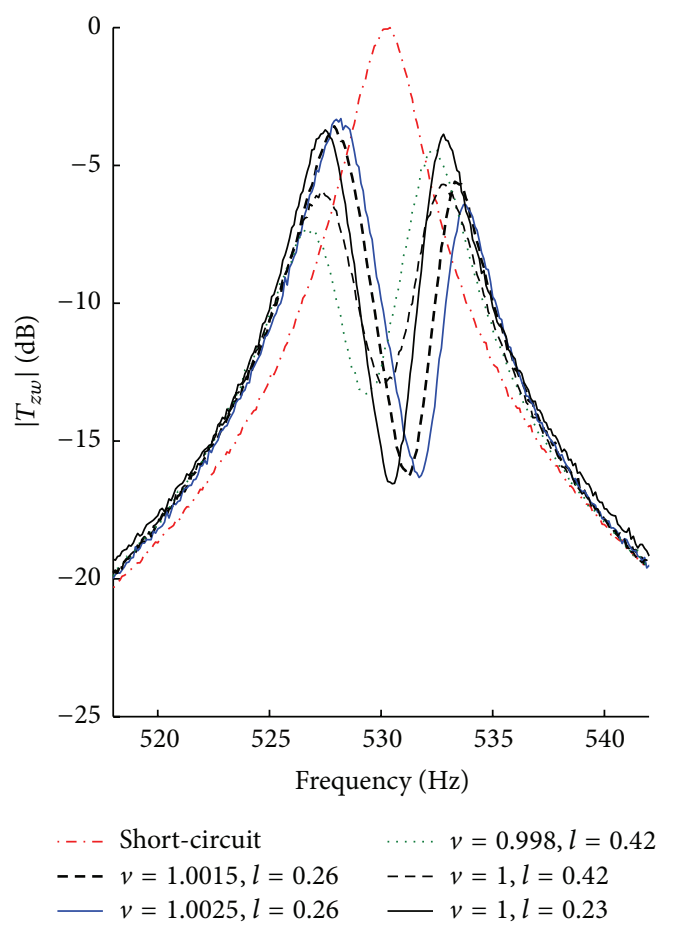

(a)

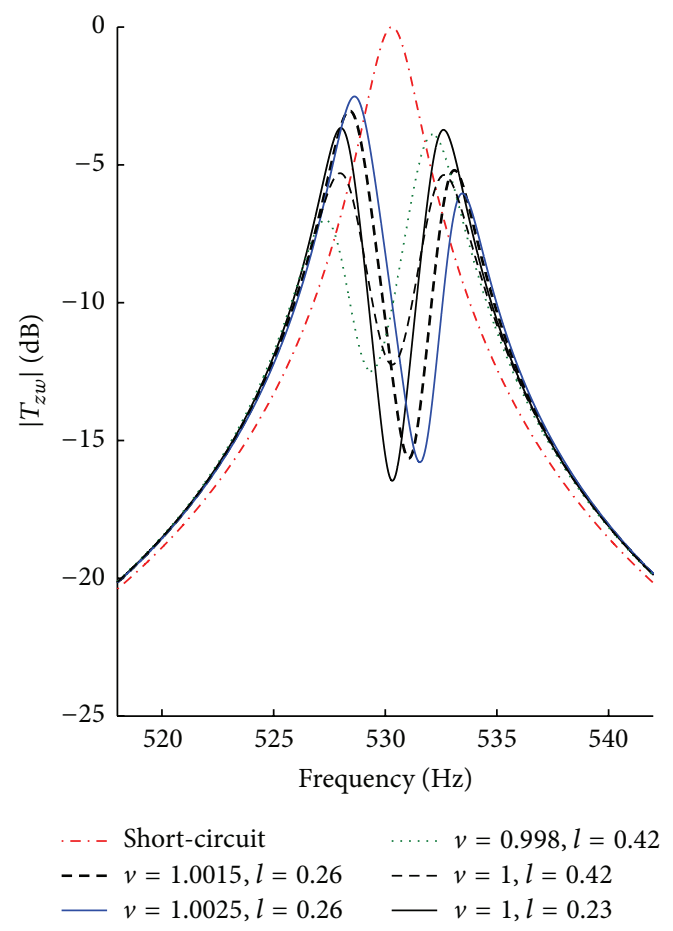

(b)

FIGURE 11: Experimental (a) and numerical (b) FRFs for the plate $\left(k_{n}=0.0081\right)$.

TABLE 6: Results of the Monte Carlo simulations for $v \neq 1$ and $l \neq 1$.

\begin{tabular}{lccc}
\hline Mean value of $\Delta[\mathrm{dB}]$ & Standard deviation of $\Delta[\mathrm{dB}]$ & Minimum value of $\Delta[\mathrm{dB}]$ & Maximum value of $\Delta[\mathrm{dB}]$ \\
\hline-0.20 & 0.46 & -2.55 & 2.36 \\
\hline
\end{tabular}

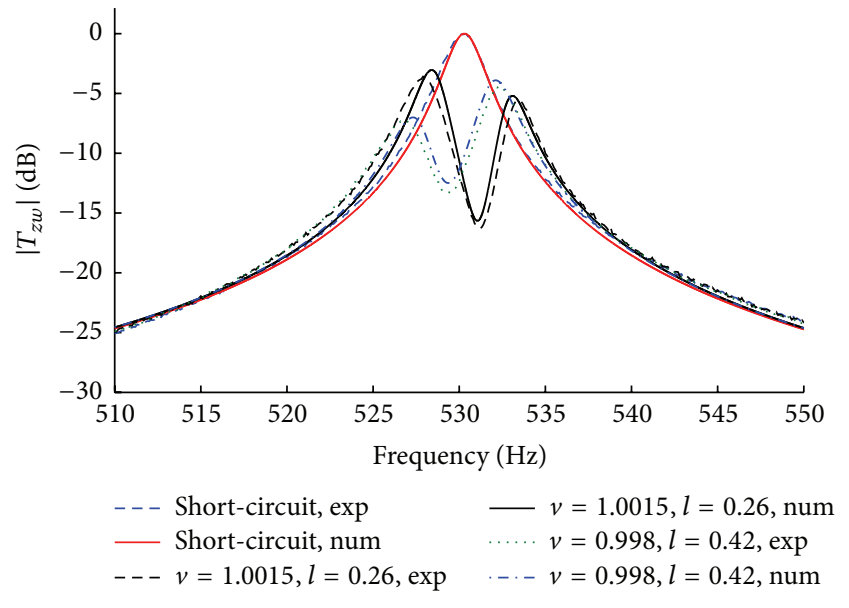

FIGURE 12: Experimental and numerical FRFs for the plate $\left(k_{n}=\right.$ $0.0081)$.

coupling coefficient exists when a perfect tuning is reached. The same linear behaviour exists when there is mistuning on either the electrical eigenfrequency or damping. Moreover, the paper indicates how the loss of attenuation essentially depends on only one bias if the electrical damping is overestimated and describes how the effects of the two bias types (on the electrical eigenfrequency and damping) combine with each other when the damping is underestimated.

This allows an approximated model to be achieved for describing the behaviour of mistuned shunt systems, which was initially validated numerically using Monte Carlo simulations and then experimentally through the use of two test structures. Furthermore, the use of overestimated resistance values is demonstrated to limit the loss of attenuation due to mistuning.

\section{Appendix}

\section{A. $T_{z w}$ Normalised Analytical Expression}

This appendix provides the mathematical process that allows (17) to be derived from (10).

Based on (10) and passing from the Laplace domain to the frequency domain (i.e., $s=j \omega$ ), the expression of $T_{z w}$ can be written as follows: 
TABLE 7: Attenuations for the mode of the plate.

\begin{tabular}{|c|c|c|c|c|c|}
\hline & & $l=0.6, v=1$ & $l=0.6, v=0.9925$ & $l=0.8, v=1.01$ & $l=1.2, v=0.998$ \\
\hline \multirow{2}{*}{$\mathrm{EA}[\mathrm{dB}]$} & $k_{n}=0.0081$ & 6.5 & 1.7 & 1.4 & 5.8 \\
\hline & $k_{n}=0.0240$ & 12.9 & 9.3 & 10.0 & 11.5 \\
\hline \multirow{2}{*}{$\mathrm{NA}[\mathrm{dB}]$} & $k_{n}=0.0081$ & 6.2 & 2.0 & 1.8 & 5.4 \\
\hline & $k_{n}=0.0240$ & 12.7 & 9.6 & 9.6 & 12.2 \\
\hline \multirow{2}{*}{$\mathrm{MA1}[\mathrm{dB}]$} & $k_{n}=0.0081$ & 6.3 & 2.4 & 2.2 & 5.5 \\
\hline & $k_{n}=0.0240$ & 12.7 & 10.1 & 10.1 & 12.5 \\
\hline \multirow{2}{*}{ MA2 [dB] } & $k_{n}=0.0081$ & 6.3 & 2.3 & 2.1 & 5.5 \\
\hline & $k_{n}=0.0240$ & 12.7 & 10.1 & 10.1 & 12.5 \\
\hline \multirow{2}{*}{ MA3 [dB] } & $k_{n}=0.0081$ & 6.4 & 2.5 & 2.3 & 5.6 \\
\hline & $k_{n}=0.0240$ & 12.8 & 10.1 & 10.1 & 12.6 \\
\hline
\end{tabular}

$$
T_{z w}\left(x_{M}\right)=\Phi_{n}\left(x_{M}\right) \Phi_{n}\left(x_{F}\right) \frac{-\omega^{2}+2 j \omega d_{i} \omega_{p}+\omega_{p}^{2}}{\left(-\omega^{2}+2 j \omega d_{i} \omega_{p}+\omega_{p}^{2}\right)\left(-\omega^{2}+2 j \omega \xi_{n} \omega_{n}+\omega_{n}^{2}\right)+\gamma \psi_{n}^{2} j \omega\left(j \omega+2 d_{i} \omega_{p}\right)} .
$$

Substituting (16) into (A.1) and using (13) and (15), the expression of $T_{z w}$ can be further modified as follows:

$$
\begin{aligned}
T_{z w}\left(x_{M}\right) & =\Phi_{n}\left(x_{M}\right) \Phi_{n}\left(x_{F}\right) \frac{-\omega^{2}+2 j \omega l d_{i}^{\mathrm{opt}} v \omega_{p}^{\mathrm{opt}}+\left(v \omega_{p}^{\mathrm{opt}}\right)^{2}}{\left(-\omega^{2}+2 j \omega l d_{i}^{\mathrm{opt}} v \omega_{p}^{\mathrm{opt}}+\left(v \omega_{p}^{\mathrm{opt}}\right)^{2}\right)\left(-\omega^{2}+2 j \omega \xi_{n} \omega_{n}+\omega_{n}^{2}\right)+\gamma \psi_{n}^{2} j \omega\left(j \omega+2 l d_{i}^{\mathrm{opt}} v \omega_{p}^{\mathrm{opt}}\right)} \\
& =\frac{\Phi_{n}\left(x_{M}\right) \Phi_{n}\left(x_{F}\right)\left[-\omega^{2}+2 j \omega l v \sqrt{\gamma \psi_{n}^{2} / 2}+v^{2}\left(\omega_{n}^{2}+\gamma \psi_{n}^{2}\right)\right]}{\left(-\omega^{2}+2 j \omega l v \sqrt{\gamma \psi_{n}^{2} / 2}+v^{2}\left(\omega_{n}^{2}+\gamma \psi_{n}^{2}\right)\right)\left(-\omega^{2}+2 j \omega \xi_{n} \omega_{n}+\omega_{n}^{2}\right)+\gamma \psi_{n}^{2} j \omega\left(j \omega+2 l v \sqrt{\gamma \psi_{n}^{2} / 2}\right)} .
\end{aligned}
$$

By dividing both the numerator and the denominator of (A.2) by a factor $\omega_{n}^{4}$, defining the nondimensional frequency $\varphi=\omega / \omega_{n}$, and using (12), the final expression of $T_{z w}$ can be obtained as follows (see (17)):

$$
\begin{aligned}
& T_{z w}\left(x_{M}\right)=\frac{\omega_{n}^{4}}{\omega_{n}^{4}} \cdot \frac{\Phi_{n}\left(x_{M}\right) \Phi_{n}\left(x_{F}\right)\left[-\omega^{2}+2 j \omega l v \sqrt{\gamma \psi_{n}^{2} / 2}+v^{2}\left(\omega_{n}^{2}+\gamma \psi_{n}^{2}\right)\right]}{\left(-\omega^{2}+2 j \omega l v \sqrt{\gamma \psi_{n}^{2} / 2}+v^{2}\left(\omega_{n}^{2}+\gamma \psi_{n}^{2}\right)\right)\left(-\omega^{2}+2 j \omega \xi_{n} \omega_{n}+\omega_{n}^{2}\right)+\gamma \psi_{n}^{2} j \omega\left(j \omega+2 l v \sqrt{\gamma \psi_{n}^{2} / 2}\right)} \\
& {\left[\Phi_{n}\left(x_{M}\right) \Phi_{n}\left(x_{F}\right) / \omega_{n}^{2}\right]\left[-\omega^{2} / \omega_{n}^{2}+\sqrt{2} j\left(\omega l v \sqrt{\gamma \psi_{n}^{2}} / \omega_{n}^{2}\right)+v^{2}\left(\omega_{n}^{2}+\gamma \psi_{n}^{2}\right) / \omega_{n}^{2}\right]} \\
& =\overline{\left(-\omega^{2} / \omega_{n}^{2}+\sqrt{2} j\left(\omega l v \sqrt{\gamma \psi_{n}^{2}} / \omega_{n}^{2}\right)+v^{2}\left(\omega_{n}^{2}+\gamma \psi_{n}^{2}\right) / \omega_{n}^{2}\right)\left(-\omega^{2}+2 j \omega \xi_{n} \omega_{n}+\omega_{n}^{2}\right)+\left(\gamma \psi_{n}^{2} \omega / \omega_{n}^{3}\right) j\left(j\left(\omega / \omega_{n}\right)+\sqrt{2}\left(l v \sqrt{\gamma \psi_{n}^{2}} / \omega_{n}\right)\right)} \\
& =\frac{\Phi_{n}\left(x_{M}\right) \Phi_{n}\left(x_{F}\right)}{\omega_{n}^{2}} \cdot \frac{-\varphi^{2}+\sqrt{2} j \varphi k_{n} l v+v^{2}\left(1+k_{n}^{2}\right)}{\left(-\varphi^{2}+\sqrt{2} j \varphi k_{n} l v+v^{2}\left(1+k_{n}^{2}\right)\right)\left(-\varphi^{2}+2 j \xi_{n} \varphi+1\right)+j \varphi k_{n}^{2}\left(j \varphi+\sqrt{2} k_{n} l v\right)} .
\end{aligned}
$$


TABLE 8: Attenuations for the mode of the beam.

\begin{tabular}{llcccc}
\hline & & $l=0.6, v=1.1$ & $l=1.5, v=1$ & $l=0.6, v=0.8$ & $l=1.2, v=0.8$ \\
\hline \multirow{2}{*}{ EA [dB] } & $k_{n}=0.2002$ & 19.3 & 23.1 & 3.1 & 13.9 \\
& $k_{n}=0.5108$ & 29.0 & 29.4 & 16.4 & 25.7 \\
\hline \multirow{2}{*}{ NA [dB] } & $k_{n}=0.2002$ & 18.6 & 22.3 & 3.9 & 13.5 \\
& $k_{n}=0.5108$ & 29.8 & 30.8 & 17.1 & 26.9 \\
\hline \multirow{2}{*}{ MA1 [dB] } & $k_{n}=0.2002$ & 19.8 & 22.3 & 4.9 & 12.9 \\
& $k_{n}=0.5108$ & 30.5 & 30.9 & 17.9 & 26.9 \\
\hline \multirow{2}{*}{ MA2 [dB] } & $k_{n}=0.2002$ & 19.8 & 22.3 & 4.8 & 12.9 \\
& $k_{n}=0.5108$ & 30.5 & 30.8 & 17.9 & 26.9 \\
\multirow{2}{*}{ MA3 [dB] } & $k_{n}=0.2002$ & 19.8 & 22.3 & 5.2 & 13.0 \\
& $k_{n}=0.5108$ & 30.5 & 30.9 & 17.9 & 26.8 \\
\hline
\end{tabular}

\section{B. att $k$ Analytical Expression}

The mathematical process used to express attk (see (21)) is explained here.

The expression of $T_{z w}$ in (17) can be rearranged by separating the real and imaginary parts at the numerator and denominator as follows:

$$
T_{z w}\left(x_{M}\right)=\frac{\Phi_{n}\left(x_{M}\right) \Phi_{n}\left(x_{F}\right)}{\omega_{n}^{2}} \cdot \frac{A_{z w}+j B_{z w}}{C_{z w}+j D_{z w}},
$$

where

$$
\begin{aligned}
A_{z w}= & -\varphi^{2}+v^{2}\left(1+k_{n}^{2}\right), \\
B_{z w}= & \sqrt{2} \varphi k_{n} l v, \\
C_{z w}= & \left(-\varphi^{2}+v^{2}\left(1+k_{n}^{2}\right)\right)\left(-\varphi^{2}+1\right) \\
& -2 \sqrt{2} \varphi^{2} k_{n} l v \xi_{n}-\varphi^{2} k_{n}^{2}, \\
D_{z w}= & \sqrt{2} \varphi k_{n} l v\left(-\varphi^{2}+1\right) \\
& +2 \xi_{n} \varphi\left(-\varphi^{2}+v^{2}\left(1+k_{n}^{2}\right)\right)+\sqrt{2} \varphi k_{n}^{3} l v .
\end{aligned}
$$

According to [23], $\left|T_{z w}\right|$ can be expressed as follows:

$$
\begin{aligned}
\left|T_{z w}\left(x_{M}\right)\right|^{2} & \\
= & \left(\frac{\Phi_{n}\left(x_{M}\right) \Phi_{n}\left(x_{F}\right)}{\omega_{n}^{2}}\right)^{2} \\
& \cdot \frac{A_{z w}^{2} C_{z w}^{2}+B_{z w}^{2} D_{z w}^{2}+B_{z w}^{2} C_{z w}^{2}+A_{z w}^{2} D_{z w}^{2}}{\left(C_{z w}^{2}+D_{z w}^{2}\right)^{2}} .
\end{aligned}
$$

The expression of (B.3) can be evaluated in $\omega=\omega_{p}=\omega_{p}^{\mathrm{opt}}$ (the case of perfect tuning is considered here), which in turn corresponds to $\varphi=\omega_{p}^{\mathrm{opt}} / \omega_{n}=\omega_{n}^{\mathrm{oc}} / \omega_{n}=\sqrt{1+k_{n}^{2}}$ :

$$
\begin{aligned}
\left|T_{z w}\left(x_{M}\right)\right|_{\omega_{p}}= & \left|\frac{\Phi_{n}\left(x_{M}\right) \Phi_{n}\left(x_{F}\right)}{\omega_{n}^{2}}\right| \\
& \cdot \frac{\sqrt{2}}{\left(k_{n}+2 \sqrt{2} \xi_{n}\right) \sqrt{1+k_{n}^{2}}} .
\end{aligned}
$$

According to [11], $\max \left(\left|G_{z w}\right|\right)=\left|\Phi_{n}\left(x_{M}\right) \Phi_{n}\left(x_{F}\right)\right| \mid$ $\left(2 \xi_{n} \omega_{n}^{2} \sqrt{1-\xi_{n}^{2}}\right)$. Therefore

$$
\text { att } k=\frac{\max \left(\left|G_{z w}\right|\right)}{\left|T_{z w}\right|_{\omega_{p}}}=\left(k_{n}+2 \sqrt{2} \xi_{n}\right) \sqrt{\frac{\left(1+k_{n}^{2}\right)}{8 \xi_{n}^{2}\left(1-\xi_{n}^{2}\right)}} .
$$

\section{List of the Symbols}

This appendix clarifies the meaning of the symbols used.

The symbol $*$ represents a generic mistuned condition.

The symbol $\sim$ represents an estimate of the considered quantity.

$A_{\mathrm{dB}}$ expresses the attenuation in decibels achieved in case of perfect tuning (i.e., $l=1, v=1$ ).

$A_{\mathrm{dB}}^{*}$ expresses the attenuation in decibels achieved in case of mistuning (this is evidenced by the asterisk).

When $A_{\mathrm{dB}}$ and $A_{\mathrm{dB}}^{*}$ are evaluated at specific points (i.e., given values of $l, v$, or $k_{n}$ ), the following expressions are used: $\left.A_{\mathrm{dB}}\right|_{k_{n}}$ and $\left.A_{\mathrm{dB}}^{*}\right|_{l=1, v=v_{x}}$, as examples. The former expression indicates that $A_{\mathrm{dB}}$ is computed in correspondence with a given value $k_{n}$ of the effective coupling factor, whereas the latter indicates that $A_{\mathrm{dB}}^{*}$ is computed for $l=1$ and $v=v_{x}$.

$\widetilde{A}_{\mathrm{dB}}^{*}$ and $\widetilde{A}_{\mathrm{dB}}$ represent the values of $A_{\mathrm{dB}}^{*}$ and $A_{\mathrm{dB}}$, respectively, which are estimated using the model proposed in the paper, that is, by (30), (32), and (37).

As for the angular coefficient $m$ and the intercept $q$ of the linear relations presented in the paper (e.g., (25), (27), (30), and (32)), when they have a subscript 0 , they are calculated for a perfectly tuned shunt impedance. Conversely, when they 
are calculated for a mistuned system, they have an $*$ as a superscript and a subscript equal to $l$ (for a mistuning on $d_{i}$ and with $\omega_{p}$ perfectly tuned) or $v$ (for a mistuning on $\omega_{p}$ and with $d_{i}$ perfectly tuned).

\section{Competing Interests}

The authors declare that there are no competing interests regarding the publication of this paper.

\section{References}

[1] A. Preumont, Vibration Control of Active Structures: An Introduction, Kluwer Academic, Dordrecht, Netherlands, 2nd edition, 2002.

[2] A. Preumont, Mechatronics-Dynamics of Electromechanical and Piezoelectric Systems, Springer, Dordrecht, The Netherlands, 2006.

[3] C. Fuller, S. Elliott, and P. Nelson, Active Control of Vibration, Acadamic Press, London, UK, 1997.

[4] N. W. Hagood and A. von Flotow, "Damping of structural vibrations with piezoelectric materials and passive electrical networks," Journal of Sound and Vibration, vol. 146, no. 2, pp. 243-268, 1991.

[5] D. Guyomar, Y. Jayet, L. Petit et al., "Synchronized switch harvesting applied to selfpowered smart systems: piezoactive microgenerators for autonomous wireless transmitters," Sensors and Actuators A: Physical, vol. 138, no. 1, pp. 151-160, 2007.

[6] J. Tang and K. W. Wang, "Active-passive hybrid piezoelectric networks for vibration control: comparisons and improvement," Smart Materials and Structures, vol. 10, no. 4, pp. 794-806, 2001.

[7] K. Yamada, H. Matsuhisa, H. Utsuno, and K. Sawada, "Optimum tuning of series and parallel LR circuits for passive vibration suppression using piezoelectric elements," Journal of Sound and Vibration, vol. 329, no. 24, pp. 5036-5057, 2010.

[8] P. Soltani, G. Kerschen, G. Tondreau, and A. Deraemaeker, "Piezoelectric vibration damping using resonant shunt circuits: an exact solution," Smart Materials and Structures, vol. 23, no. 12, Article ID 125014, 2014.

[9] J. Høgsberg and S. Krenk, "Balanced calibration of resonant shunt circuits for piezoelectric vibration control," Journal of Intelligent Material Systems and Structures, vol. 23, no. 17, pp. 1937-1948, 2012.

[10] J. Høgsberg and S. Krenk, "Balanced calibration of resonant piezoelectric RL shunts with quasi-static background flexibility correction," Journal of Sound and Vibration, vol. 341, pp. 16-30, 2015.

[11] O. Thomas, J. Ducarne, and J.-F. Deü, "Performance of piezoelectric shunts for vibration reduction," Smart Materials and Structures, vol. 21, no. 1, article 015008, 2012.

[12] E. Doebelin, Measurement Systems: Application and Design, McGraw-Hill, New York, NY, USA, 2003.

[13] J. J. Hollkamp and T. F. Starchville Jr., "Self-tuning piezoelectric vibration absorber," Journal of Intelligent Material Systems and Structures, vol. 5, no. 4, pp. 559-566, 1994.

[14] B. Zhou, F. Thouverez, and D. Lenoir, "Essentially nonlinear piezoelectric shunt circuits applied to mistuned bladed disks," Journal of Sound and Vibration, vol. 333, no. 9, pp. 2520-2542, 2014.
[15] B. Zhou, F. Thouverez, and D. Lenoir, "Vibration reduction of mistuned bladed disks by passive piezoelectric shunt damping techniques," AIAA Journal, vol. 52, no. 6, pp. 1194-1206, 2014.

[16] S. Behrens, A. J. Fleming, and S. O. R. Moheimani, "A broadband controller for shunt piezoelectric damping of structural vibration," Smart Materials and Structures, vol. 18, no. 12, pp. 1828, 2003.

[17] D. Niederberger, M. Morari, and S. Pietrzko, "Adaptive resonant shunted piezoelectric devices for vibration suppression," in Proceedings of the Smart Structures and Materials 2003: Smart Structures and Integrated Systems, pp. 213-224, San Diego, Calif, USA, March 2003.

[18] H. Hanselka, "Adaptronik und fragen zur systemzuverl ATP," Automatisierungstechnische Praxis, vol. 2, pp. 44-49, 2002.

[19] M. Berardengo, A. Cigada, S. Manzoni, and M. Vanali, "Vibration control by means of piezoelectric actuators shunted with LR impedances: performance and robustness analysis," Shock and Vibration, vol. 2015, Article ID 704265, 30 pages, 2015.

[20] S. Moheimani and A. Fleming, Piezoelectric Transducers for Vibration Control and Damping, Springer, London, UK, 2006.

[21] S. O. R. Moheimani, A. J. Fleming, and S. Behrens, "On the feedback structure of wideband piezoelectric shunt damping systems," Smart Materials and Structures, vol. 12, no. 1, pp. 49$56,2003$.

[22] B. De Marneffe and A. Preumont, "Vibration damping with negative capacitance shunts: theory and experiment," Smart Materials and Structures, vol. 17, no. 3, article 035015, 2008.

[23] S. Krenk, "Frequency analysis of the tuned mass damper," Journal of Applied Mechanics, vol. 72, no. 6, pp. 936-942, 2005.

[24] D. J. Ewins, Modal Testing: Theory, Practice and Application, Research Studies Press, Baldock, UK, 2nd edition, 2000.

[25] J. Ducarne, O. Thomas, and J.-F. Deü, "Placement and dimension optimization of shunted piezoelectric patches for vibration reduction," Journal of Sound and Vibration, vol. 331, no. 14, pp. 3286-3303, 2012.

[26] O. Thomas, J.-F. Deü, and J. Ducarne, "Vibrations of an elastic structure with shunted piezoelectric patches: efficient finite element formulation and electromechanical coupling coefficients," International Journal for Numerical Methods in Engineering, vol. 80, no. 2, pp. 235-268, 2009.

[27] M. Berardengo, O. Thomas, C. Giraud-Audine, and S. Manzoni, "Improved resistive shunt by means of negative capacitance: new circuit, performances and multi-mode control," Smart Materials and Structures, vol. 25, no. 7, Article ID 075033, 2016.

[28] B. Peeters, H. Van Der Auweraer, P. Guillaume, and J. Leuridan, "The PolyMAX frequency-domain method: a new standard for modal parameter estimation?" Shock and Vibration, vol. 11, no. 3-4, pp. 395-409, 2004.

[29] O. Thomas, C. Touzé, and A. Chaigne, "Asymmetric nonlinear forced vibrations of free-edge circular plates. Part II: experiments," Journal of Sound and Vibration, vol. 265, no. 5, pp. 1075-1101, 2003.

[30] A. Brandt, Noise and Vibration Analysis-Signal Analysis and Experimental Procedures, John Wiley \& Sons, New York, NY, USA, 2011.

[31] C. H. Park and D. J. Inman, "Enhanced piezoelectric shunt design," Shock and Vibration, vol. 10, no. 2, pp. 127-133, 2003.

[32] B. Seba, J. Ni, and B. Lohmann, "Vibration attenuation using a piezoelectric shunt circuit based on finite element method analysis," Smart Materials and Structures, vol. 15, no. 2, pp. 509517, 2006. 


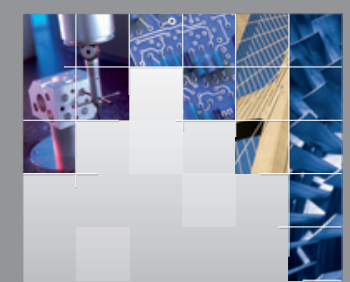

\section{Enfincering}
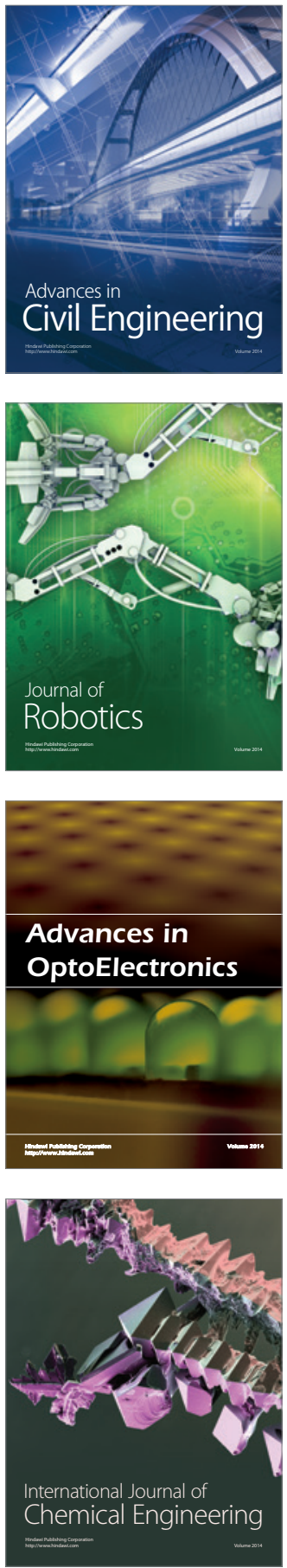

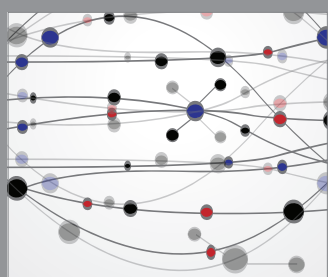

The Scientific World Journal

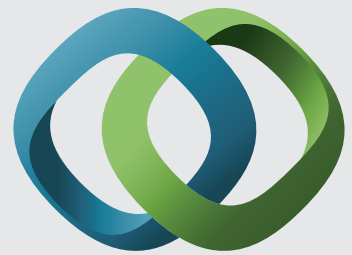

\section{Hindawi}

Submit your manuscripts at

http://www.hindawi.com
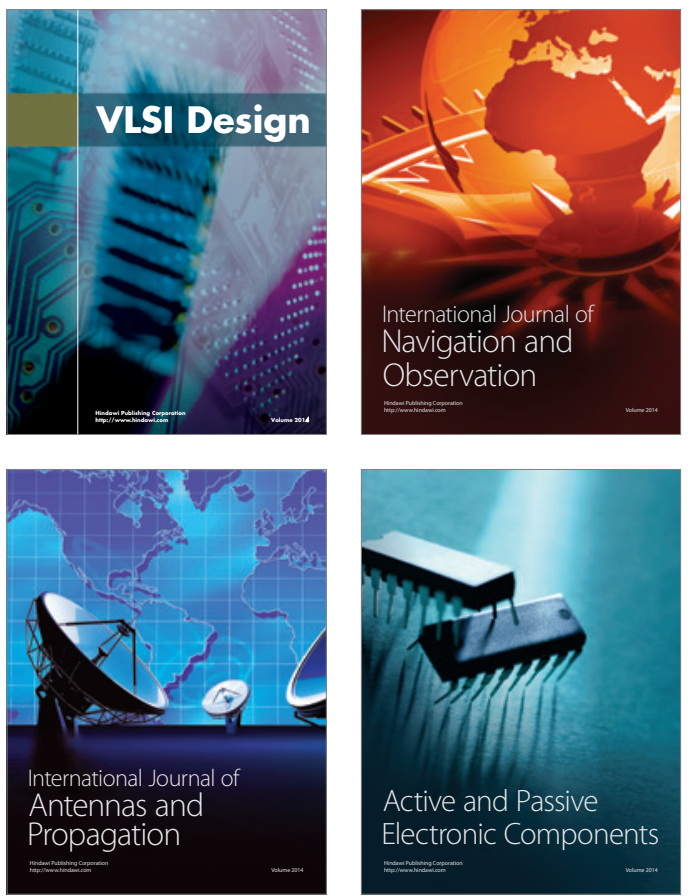
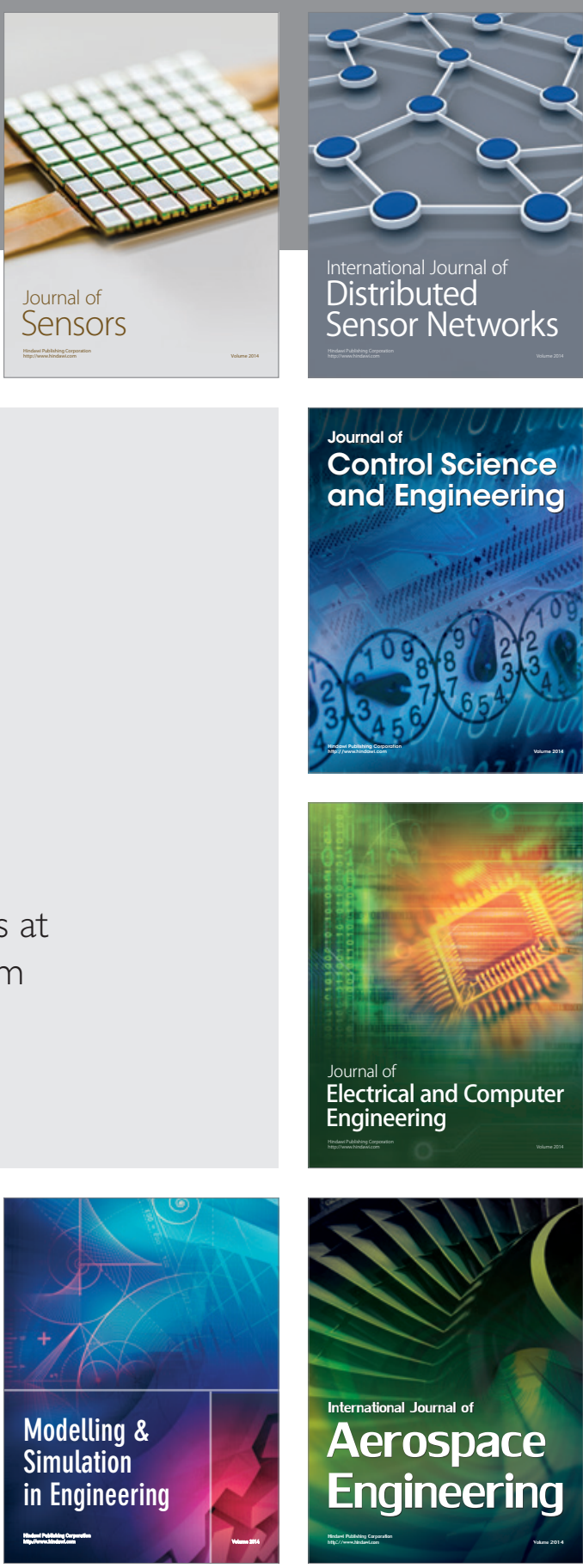

International Journal of

Distributed

Sensor Networks

Journal of

Control Science

and Engineering
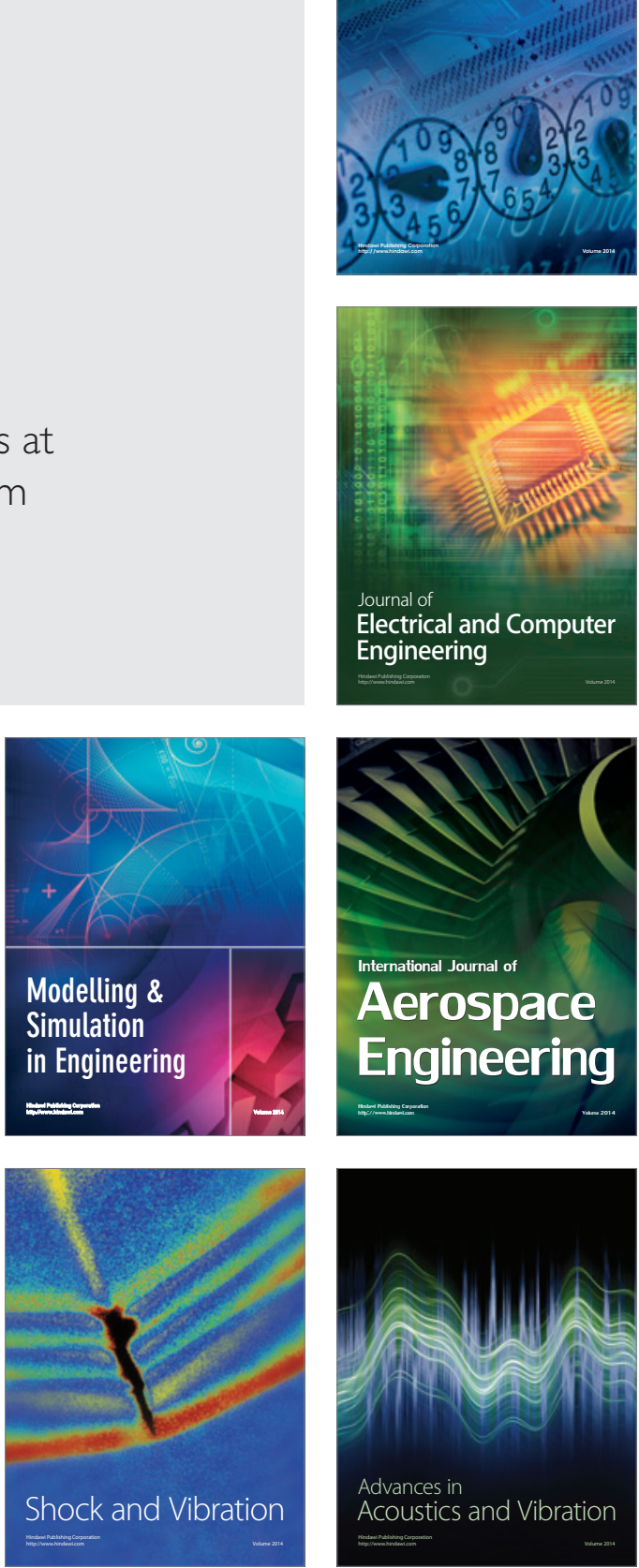